\title{
A SELF-HELP BEHAVIORAL ACTIVATION \\ TREATMENT FOR GERIATRIC \\ DEPRESSIVE SYMPTOMS
}

by

KATHRYN SARA MOSS

\section{A DISSERTATION}

Submitted in partial fulfillment of the requirements for the degree of Doctor of Philosophy in the Department of Psychology in the Graduate School of

The University of Alabama

TUSCALOOSA, ALABAMA 
Copyright Kathryn Sara Moss 2009 ALL RIGHTS RESERVED 


\begin{abstract}
The present study investigated behavioral activation (BA) bibliotherapy as a treatment for late-life depressive symptoms. BA bibliotherapy was administered using Addis and Martell's (2004) Overcoming Depression One Step at a Time as a stand-alone treatment that was completed by participants over a 4-week period. Results of an immediate intervention group were compared with those of a delayed treatment control group and treatment response for both groups was evaluated at 1-month follow-up. Results showed that symptoms on a clinician-rated measure of depression were significantly lower at post-treatment for those who received immediate BA bibliotherapy compared with those who were in the delayed treatment control condition. However, differences between groups at this period on a self-reported measure of depressive symptoms were not significant. Within-subjects analyses examining both treatment groups combined showed that clinician-rated depressive symptoms significantly decreased from pre-treatment to both post-treatment and 1-month follow-up. Self-reported depressive symptoms were significantly lower, and self-reported engagement in pleasant events was significantly higher, from pre-treatment to 1-month follow-up. These findings suggest that BA may be useful in treating mild or subthreshold depressive symptoms in an older adult population.
\end{abstract}




\section{LIST OF ABBREVIATIONS AND SYMBOLS}

$\alpha \quad$ Cronbach's index of internal consistency

$d \quad$ Cohen's $d$ : An effect size measure representing the standardized difference between two means

$d f \quad$ Degrees of freedom: number of values free to vary after certain restrictions have been placed on the data

$F \quad$ Fisher's $F$ ratio: A ratio of two variances

$M \quad$ Mean: the sum of a set of measurements divided by the number of measurements in the set

$N \quad$ Statistical notation for total sample size

$n \quad$ Statistical notation for subsample size

$\eta_{\mathrm{p}}{ }^{2} \quad$ Partial eta-squared: the proportion of total variability attributable to a factor

$p \quad$ Probability associated with the occurrence under the null hypothesis of a value as extreme as or more extreme than the observed value

$R^{2} \quad$ R-squared: the proportion of variability in a data set that is accounted for by a statistical model

$r \quad$ Pearson product-moment correlation

$S D \quad$ A statistical measure of variability in a set of data; the square root of the variance

$t \quad$ Computed value of a $t$ test

$<\quad$ Less than

$=\quad$ Equal to 


\section{ACKNOWLEDGMENTS}

I would like to express my sincerest gratitude to a number of individuals who have sustained me throughout both graduate school as well as the dissertation process. I thank Forrest Scogin so very much for providing mentorship and guidance throughout my career as a graduate student, and for doing so while maintaining a wonderful sense of humor. I also convey many thanks to my dissertation committee members, Sheila Black, Martha Crowther, Jeri Dunkin, and Tom Ward, whose input and expertise helped make this project possible. I am indebted to fellow Scogin lab members Avani Shah and Martin Morthland for providing recruitment assistance and serving as interviewers for the study, and Andrew Presnell for providing administrative support. I would also like to thank Brian Davis who originally served as an undergraduate R. A. for course credit and later offered assistance out of the goodness of his heart. The recruitment phase of this project would have been much lengthier if not for the commitment of Sharon Hamilton, Senior Housing Director, as well as Kristine Bradford, Martha Paulson, and Sam Sardi, Service Coordinators, of Lutheran Social Services in Jamestown, NY. Additionally, I am eternally grateful for the moral support and consultation provided during the best and worst of times by my intern cohort, graduate student colleagues, and close friends. I also express gratitude to my parents, Michael and Laura Moss, for encouraging me to always press on despite the challenges that have accompanied my graduate pursuits. I am indebted also to my partner James for his undying support throughout the entire graduate school process and our multiple cross-country relocations. Finally, I wish to thank the participants who graciously volunteered their time to make this research possible. 


\section{CONTENTS}

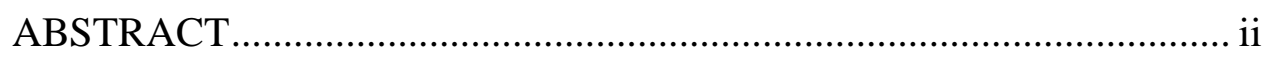

LIST OF ABBREVIATIONS AND SYMBOLS ................................ iii

ACKNOWLEDGMENTS ......................................................... iv

LIST OF TABLES ............................................................... vi

LIST OF FIGURES ............................................................... vii

1. INTRODUCTION .................................................................. 1

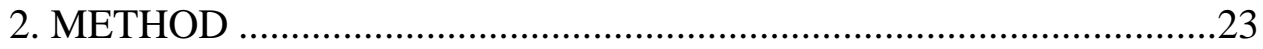

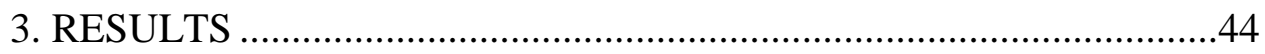

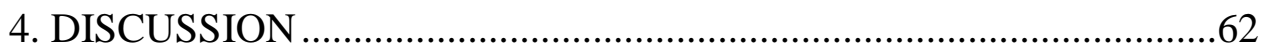

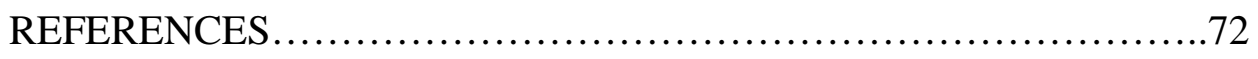

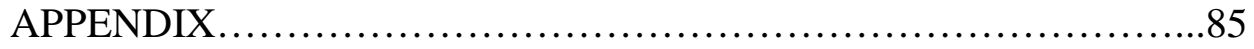




\section{LIST OF TABLES}

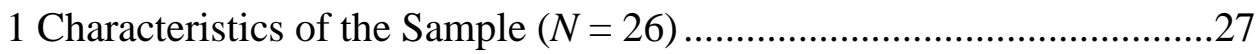

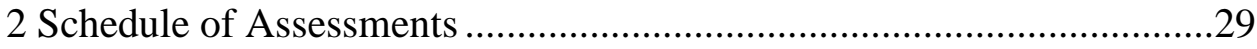

3 Raw Means of Dependent Variables and Covariates by Group for ANCOVA Analyses...........................................50

4 Between-Group ANCOVAs Examining Outcome Measures at T2

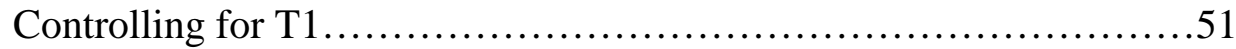

5 Pre-treatment, Post-treatment, and 1-month Follow-up Means for Within-Subjects ANOVAs......................................51

6 Pre- and Post-treatment and 1-month Follow-up Means for WithinSubjects ANOVAs.............................................53 


\section{LIST OF FIGURES}

1 The BA model of depressed affect as presented by Martell et al., (2001)..............................................................

2 Design and procedures of the immediate intervention and delayed treatment groups

3 The study enrollment process with respect to stratification..................40

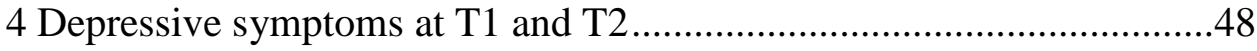

5 Significant within-subjects main effects for Time on both the HRSD

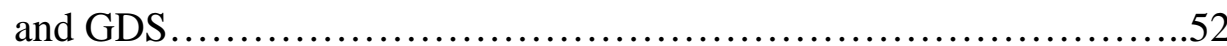

6 Significant within-subjects main effects for Time on the PES-E-20,

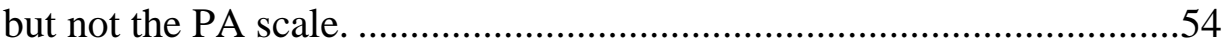

7 Somatic and Affective Symptoms at T1 and T2 on the HRSD ..............57 


\section{CHAPTER 1}

\section{INTRODUCTION}

Depression is one of the most commonly occurring mental health disorders in late life. It is widely recognized as a condition that has extensive ramifications in a variety of domains, including social relationships, health, and personal and societal finance. Rates of Major Depressive Disorder (MDD) in the older adult population are quite variable and depend largely on the level of skilled care required by those being assessed. For instance, MDD is evidenced in approximately 3-5\% of community dwelling elders (Bruce, McAvay, \& Raue, 2002). However, this percentage increases to roughly $20 \%$ of residents in skilled nursing facilities (Jones, Marcantonio, \& Rabinowitz, 2003). Rates of major depression in older adults who receive primary or home health care services or who reside in assisted living facilities fall within these bounds (e.g., Bruce et al., 2002; Watson, Garrett, Sloane, Gruber-Baldini, \& Zimmerman, 2003). Indeed, major depression occurs frequently in the older adult population. However, minor depression, dysthymia, and significant depressive symptoms occur even more frequently among individuals in this age bracket. In fact, older adults experience these subthreshold syndromes at rates comparable to, and perhaps higher than, younger persons (Blazer, 2002).

It has been suggested that current estimates of depressive disorders in the older adult population may be low, due to the fact that our existing diagnostic techniques for psychiatric disorders, particularly depressive disorders, may not take into consideration differential presentations of syndromes exhibited by older adults (e.g., Jeste, Blazer, \& First, 2005). For example, older adults are more likely to present with somatic and cognitive complaints in lieu of 
affective symptoms (e.g., depressed mood), which are more often reported by younger people with depression (Birrer \& Vemuri, 2004; Gottfries, 1998).

Depression can be particularly disabling when experienced in older adulthood. Although the disorder results in serious consequences for individuals of any age group, the effects of depression can be magnified when experienced in older adulthood due to a number of factors (e.g., decline in reserve capacity, fewer financial resources, comorbid health conditions, etc.). Depressive disorders accelerate the disease process of several health conditions common to older adults (van Gool et al., 2005), increase the risk of hospitalization (e.g., Rumsfeld et al., 2005), and predict nursing home admission (Harris \& Cooper, 2006). Depression is also associated with mortality, both as an independent risk factor and in terms of its relation to suicide. In fact, suicide rates among older adults are higher as a rule than they are for other age groups (e.g., Manthorpe \& Iliffe, 2006).

Older adults, who often live on a fixed income, may also find it challenging to manage the costs associated with depression. Direct and indirect costs of the disorder are estimated to be at least \$43 billion annually in the United States alone (Hirschfeld et al., 1997). Per person with a depressive disorder, it is estimated that direct costs (e.g., from medical visits, medication, transportation, social services) average from $\$ 1000$ - \$2500 and indirect costs (e.g., decreased work productivity, absenteeism, premature death) range from approximately $\$ 2000$ - $\$ 4000$ annually (Luppa, Heinrich, Angermeyer, König, \& Riedel-Heller, 2007).

Clearly, the negative impact of depressive spectrum disorders on the older adult population is far-reaching. As this population expands throughout the coming decades, it is likely that the numbers adversely affected by depression will increase. Therefore, it is imperative that treatments that respond well to the needs of the aging be identified, evaluated, and implemented. 


\section{Evidence-Based Treatment of Geriatric Depression}

Many mental health professionals have suggested limiting the use of psychotherapies for depression and other mental health disorders to only those treatments that have demonstrated efficacy in randomized controlled trials (Westen \& Morrison, 2001). This concept, otherwise known as evidence-based practice, came into the forefront in response to reports that medication should be used as a first-line treatment for disorders such as panic and depression (e.g., DeRubeis, Gelfond, Tang, \& Simons, 1999). While it was clinically recognized at the time of these reports that psychological interventions were available to treat such disorders, limited empirical evidence existed to support the use of these treatments (Westen \& Morrison, 2001). Therefore, proponents of evidence-based practice began developing procedures, backed by the American Psychological Association (APA), to define those treatments that were empirically supported. Recently, APA released a comprehensive policy statement on evidence-based practice in psychology (2006) that discusses the importance of integrating clinical expertise with the best research evidence in the context of patient characteristics, culture, and preferences when considering the use of certain treatments.

Scogin and McElreath (1994) conducted an investigation evaluating the efficacy of psychosocial interventions (including bibliotherapy) for geriatric depression. The authors performed a meta-analysis of 17 studies and found that the overall mean effect size for treatment versus no treatment or a placebo control was .78. In addition, the interventions demonstrated a slightly greater mean effect size for subclinical varieties of depression (.79) over major depression (.76).

A more recent meta-analysis of 18 studies was conducted examining the results from only randomized controlled trials in the treatment of late-life depression (Cuijpers, van Straten, 
$\&$ Smit, 2006). Investigators found an overall mean effect size of .72 (95\% CI: .57 - .87) for treatment versus mainly waitlist or usual care control conditions. These meta-analyses, along with other similar investigations (e.g., Engels \& Vermey, 1997; Pinquart \& Sorensen, 2001), provide evidence that psychosocial interventions are effective in general in the treatment of geriatric depression and depressive symptoms.

In addition to the examination of treatment effects in general, Scogin and colleagues (2005) have identified specific psychological treatments as evidence-based, using criteria defined by a taskforce of the Society of Clinical Psychology, in the treatment of geriatric depression. These treatments include behavioral, cognitive-behavioral, problem-solving, reminiscence, and brief psychodynamic psychotherapies as well as cognitive bibliotherapy (Scogin, Welsh, Hanson, Stump, \& Coates, 2005).

Pharmacotherapies (particularly tricyclic antidepressants (TCAs) and selective serotonin reuptake inhibitors (SSRIs)) have been identified as evidence-based interventions for late-life depression as well (e.g., Shanmugham, Karp, Drayer, Reynolds, \& Alexopoulos, 2005). SSRIs are thought to produce side effects that are less threatening to older adults than those associated with TCAs (Shanmugham et al., 2005). The use of antidepressant medication is more likely to be recommended by medical professionals as a treatment for older adults when depression is severe (Shanmugham et al., 2005).

Hollon and colleagues (2005) have noted that although antidepressant medication is effective and prevents symptom return during the course of treatment, it has little if any effectiveness in reducing the risk of relapse once an individual discontinues taking the medication. On the other hand, there is evidence that cognitive-behavioral therapy (CBT) can reduce subsequent risk following treatment, making it potentially less costly over time than 
pharmacotherapy (Hollon et al., 2005). A recent controlled research investigation found that a physician-delivered prescription for cognitive-behavioral bibliotherapy (reading self-help literature based on cognitive-behavioral theory) for depression was just as effective as standard care after a six-week intervention phase (Naylor, 2008). This finding, paired with recent identification of cognitive bibliotherapy as evidence-based, offers practitioners another empirically based option to suggest to their depressed patients which may prove valuable in overcoming treatment barriers such as cost.

\section{Bibliotherapy}

Bibliotherapy is a self-administered treatment that requires an individual to read a selfhelp book to alleviate a particular psychological problem. This form of treatment has evidenced success in treating a variety of conditions such as depression, mild alcohol abuse, and anxiety disorders (Mains \& Scogin, 2003). It has also exhibited favorable outcomes across age groups (e.g., Ackerson, Scogin, McKendree-Smith, \& Lyman, 1998; Scogin, Bynum, Stevens, \& Calhoon, 1990).

There are several ways in which bibliotherapy and traditional individual psychotherapy are fundamentally alike. For instance, they ideally both actively employ clinical methods based on scientifically and/or clinically established principles in an effort to improve client well being. To this end, both intervention methods generally raise clients' awareness of precipitants or triggers of problematic behavior or psychopathology. Many also encourage the development of strategies to effectively modify personal factors (e.g., behaviors, thoughts, emotions, etc.) that may be perpetuating psychological problems.

There are also some key differences between these two forms of treatment. For example, unlike individual psychotherapy, in which the clinician meets with the client in person once or 
twice per week for a specified amount of time (usually about an hour), bibliotherapy can be conducted as an entirely self-directed activity that can be undertaken at the client's own pace. Also, although clinicians may suggest readings and written exercises as adjuncts to traditional psychotherapy to reinforce what is discussed in session, such activities are the chief treatment methods employed in bibliotherapy. Further, in individual psychotherapy, clients have the opportunity to receive therapeutic intervention (e.g., education, feedback, problem-solving strategies, etc.) directly from the clinician and ask questions as they arise. In contrast, with selfdirected treatment, the client is required to actively engage in written materials and exercises and then draw from that information to interpret the actions necessary to resolve a particular issue on their own. The latter endeavor may be viewed by some as a more difficult task for a client to undertake. However, this difficulty is tempered by an inherent benefit of bibliotherapy; the ability of the client to read through and process the available material as many times as is necessary to properly receive and act on the information provided. It is less feasible in individual psychotherapy for the therapist to go over a particular topic or strategy in significant depth over several sessions when there are often several other matters to focus on in the time allotted.

In terms of efficacy, psychotherapy and bibliotherapy have both been found to be superior to no treatment at all (e.g., den Boer, Wiersma, \& Van Den Bosch, 2004; Scogin et al., 1990; Smith \& Glass, 1977). In studies investigating the treatment of late-life depression, the results of bibliotherapy versus psychotherapy have been relatively comparable (e.g., Cuijpers et al., 2006; Floyd, Scogin, McKendree-Smith, Floyd, \& Rokke, 2004; Floyd et al., 2006). den Boer and colleagues (2004) argue, based on the results of their meta-analysis focusing on selfdirected treatments of emotional disorders, that bibliotherapy exhibits robust effects and more research attention should be directed toward integrating it into medical practice. Others suggest 
that the duration of treatment is important such that bibliotherapy may be as effective as shortterm treatment (i.e., 4 - 20 weeks) that is provided by a professional, but may potentially not be as effective as professional treatment of a longer duration (den Boer et al., 2004).

In terms of long-term effectiveness, follow-up studies on bibliotherapy investigations have found that, largely, symptom improvement is maintained (e.g., Floyd et al., 2006; Jamison \& Scogin, 1995; Landreville, 1998; McKendree-Smith, 2000). In addition, bibliotherapy has comparable negative response rates to those of therapist-administered treatments (Scogin et al., 1996). However, some research suggests that bibliotherapy may be associated with greater recurrence rates of depression at two-year follow-up (Floyd et al., 2006).

Several benefits of self-administered treatments have been noted. First, they are costeffective. Purchasing a book or workbook results in only a fraction of the cost required for regular medication use or several sessions of psychotherapy. If used as a stand-alone treatment, bibliotherapy could be initiated by the individual or even suggested by a health care professional such as a nurse or primary care provider without incurring significant additional healthcare costs. Bibliotherapy can help reach those who are unable to afford the more traditional, expensive forms of treatment. Another benefit of bibliotherapy is the ability to reach and treat a broader scope of individuals, who may be limited in their ability or willingness to engage in more traditional types of treatment due to stigma, illness or functional impairment, transportation difficulties, or other issues especially relevant to older adults. Also, for those who have limited time or may be on an alternate schedule, an independent treatment such as bibliotherapy can be undertaken at the individual's convenience and at his or her own pace. It is also thought that the self-administered nature of bibliotherapy lends itself nicely to heightening an individual's sense of treatment-related self-efficacy and responsibility (e.g., Floyd, 2003). This increased 
motivation and responsibility may be pivotal in ensuring the individual enacts the treatment techniques suggested in the literature and may potentially enhance therapeutic outcomes.

Despite the many benefits associated with bibliotherapy, it is not appropriate for everyone. First, there are practical barriers, such as blindness or visual impairment, to participation in traditional bibliotherapy. Fortunately, if visual acuity is a problem, some adaptations can be made to facilitate reading. For example, large print materials or magnifying glasses can be provided. The inability to read is also a pragmatic issue. Those who are unable to read should be considered for more appropriate treatments, such as individual psychotherapy. Finally, patient preference should also be considered to determine whether bibliotherapy is even of interest to the individual in need of treatment.

Floyd (2003) also pointed out that individuals with a fear of evaluation at tasks resembling schoolwork or who tend to perform below average on academic undertakings may not be well-suited for bibliotherapy. These individuals may be more resistant toward the independent tasks required as a part of bibliotherapy, such as lengthy readings or written activities, which may be viewed as "homework," (Floyd, 2003).

In addition to the best persons for which bibliotherapy may be suited, there may also be certain situations under which this method of treatment is ideal. Mains and Scogin (2003) have provided a set of practice recommendations designed to aid practitioners in determining the circumstances under which a self-administered treatment may be most appropriate and effective. These guidelines are as follows: 1) the client should not be declining as they progress through the therapy - as such, individual progress and treatment response should be monitored; 2) including a maintenance program for those who respond well to treatment may result in more stable, longlasting gains; 3) individual client characteristics (e.g., severity of symptoms, motivational issues, 
etc.) should be considered before implementing bibliotherapy; 4) the incorporation of additional stimuli (e.g., telephone 'check-ins,' audiotapes, etc.) tends to result in improved outcomes; 5) some level of therapist contact also leads to better treatment results; 6) the use of bibliotherapy for the disorder one is attempting to treat (especially if contact with the therapist is limited) should have sufficient research support; 7) self-help may not be appropriate for habit control problems (e.g., smoking cessation); 8) even in the context of everyday application, clinicians should be wary of administering self-help programs without at least some form of minimal contact; 9) only a few self-help books have been tested for efficacy; and 10) those who choose to recommend self-administered treatment to clients should consider materials that have undergone rigorous testing. This comprehensive set of guidelines provides a good foundation from which clinicians and other healthcare professionals can make decisions about the applicability of bibliotherapy to various clients in various situations.

Recent research on a cognitive-behavioral bibliotherapy program for depression in younger and middle-aged adults offers additional evidence to support the aforementioned recommendations regarding the use of some level of therapist contact during self-directed treatment. In this research, participants in treatment groups who received weekly 5- or 30-minute phone contacts during the treatment phase obtained significantly greater improvements in depressive symptoms than those in a book-only control group (Bilich, Deane, Phipps, Barisic, \& Gould, 2008). Interestingly, Bilich et al. (2008) found that length of therapist contact was irrelevant such that change in depressive symptoms over the course of the investigation did not differ significantly between the 5- and 30-minute contact groups. This suggests that even very minimal phone contact can boost treatment response in bibliotherapy. 
Redding and colleagues (2008) agree with the recommendation that the use of self-help treatments should be driven by empirical evidence. However, they assert that this evidence should be based not only on studies of the effectiveness of individual texts, but also in terms of whether the provision of guidance in the texts is consistent with current psychological science. Using a scale derived from literature on the evaluation of self-help books and the characteristics that contribute to their effectiveness (i.e., overall usefulness, grounding in psychological science, extent to which the program offers reasonable expectations, extent to which the program offers specific guidance for implementing self-help techniques and monitoring treatment progress, and whether the program offers potentially harmful advice), expert ratings of the 50 top-selling selfhelp books for anxiety, depressive, and trauma-related disorders were conducted (Redding, Herbert, Forman, \& Guadiano, 2008). Results of this investigation indicated that the following characteristics of the books or authors were associated with higher ratings: 1) presence of a cognitive-behavioral perspective; 2) written by mental health professionals; 3) written by authors holding a doctoral degree; and 4) those focusing on specific problems (Redding et al., 2008).

\section{Bibliotherapy for Older Adult Depression}

Bibliotherapy has proven successful in treating depression across the lifespan. As mentioned previously, cognitive bibliotherapy, or the reading of a self-help presentation of cognitive therapy (Burns, 1980), has been identified as an evidence-based treatment for geriatric depression. Most of the studies supporting this treatment have used the popular Feeling Good text by Burns (1980). It is possible that other books based on cognitive theory would yield similar results in the treatment of late-life depression. However, limited research has been conducted in this area and thus this notion has yet to be confirmed. Further, despite not being deemed as evidence-based due to a lack of studies at this time from which to corroborate 
evidence, behavioral bibliotherapy has shown promising results as a treatment for geriatric depression. Behavioral bibliotherapy is defined as the reading of a self-help presentation of behavior therapy (Lewinsohn, Munoz, Youngren, \& Zeiss, 1986). In a study conducted by Scogin, Jamison, and Gochneaur (1989), behavioral bibliotherapy using Lewinsohn and colleagues' (1986) Control Your Depression, when compared with Burns' cognitive bibliotherapy, yielded non-differentially efficacious results. Again, it is possible that other books based on behavioral theory (as well as other theories) would produce positive outcomes in the treatment of depressive disorders, but there is a lack of research in this area at present to support this idea.

In studies examining older adults' preferences for psychological and pharmacological treatments for depression (Choi \& Morrow-Howell, 2007; Landreville, Landry, Baillargeon, Guérette, \& Matteau, 2001), findings revealed that cognitive bibliotherapy (in addition to cognitive therapy) was preferred over antidepressant medication when depressive symptoms were mild to moderate. Other studies looking at the treatment preferences of older adults found that they tend to be just as (or even more) accepting of psychotherapeutic interventions as they are for pharmacotherapies for depression (e.g., Gum et al., 2006; Rokke \& Scogin, 1995). This indicates that older adults may be looking for, and are often more accepting of, nonpharmacological treatments for depressive disorders. Bibliotherapy is a viable treatment option for older adults with a preference toward non-pharmacological interventions.

Despite these findings, it should be noted that acceptability of treatment type might differ based on the population of older adults being studied. A recent study investigating low-income older adults' acceptance of depression treatments found that although homebound older adults were accepting of both antidepressant medication and cognitive bibliotherapy as depression 
treatments, they held significantly less favorable attitudes toward these therapies than did their ambulatory peers (Choi \& Morrow-Howell, 2007). These results should be considered in research investigating homebound older adult samples.

Also, despite the fact that self-administered treatments generally result in positive outcomes when used by older adults, Floyd (2003) described some 'depression-specific caveats' when using bibliotherapy as a stand-alone treatment with this population. First, cognitive impairment can contribute to limited responsiveness to the treatment. This not only refers to memory impairment, but also attentional issues (Floyd, 2003). Further, Floyd (2003) points out that individuals who have motivational or behavioral initiation issues would not be good candidates for bibliotherapy, as the nature of the intervention necessitates that the participant take charge of his or her own treatment. Finally, those who do not respond well to psychotherapy will also likely not respond well to bibliotherapy. Results of one study indicated that participants who failed to respond to a bibliotherapy intervention also failed to respond to 16 sessions of individual cognitive psychotherapy (Floyd, 2003).

Again, it is important to keep in mind that the majority of the findings related to bibliotherapy are based on cognitive bibliotherapy and, in general, one or two specific texts. There is no empirical evidence at this time that generalizes these findings to other treatments or even other readings under the same treatment domain. However, clinical speculation might lead one to hypothesize that the same findings might be generalizable to other self-administered treatments.

\section{Behavioral Activation Therapy and Bibliotherapy}

The literature on behavioral bibliotherapy treatment has remained quite limited despite the fact that it has yielded promising results in the treatment of geriatric depression. Addis and 
Martell's (2004) Overcoming Depression One Step at a Time has emerged as one of the latest developments in the self-help literature for depression. This workbook is based on a behavioral treatment, behavioral activation therapy.

Behavioral activation, or BA, (Jacobson, Martell, \& Dimidjian, 2001) is a focused method of targeting activity level that has been gaining much support in the literature as a treatment for depression (e.g., Dimidjian et al., 2006; Jacobson et al., 1996; Porter, Spates, \& Smitham, 2004). BA emerged from cognitive therapy as a stand-alone treatment for depression after results from a groundbreaking investigation of the components responsible for change in cognitive therapy indicated that the behavioral components alone resulted in just as much change as the entire therapeutic program in young and middle-aged adults (Jacobson et al., 1996). These behavioral components are rooted in learning theory models of depression, which suggest that increasing positively reinforcing behaviors can lead to more positive consequences and thus contribute to improvements in mood (e.g. Ferster, 1973). Other behavioral treatment models that have followed this approach (e.g., Lewinsohn \& Graf, 1973) have focused on increasing pleasant events as a means to facilitate positive reinforcement. Although clients in BA are encouraged to increase pleasant events, they are instructed to do so while keeping the functional context of everyday life carefully in mind, and not solely for the sake of increasing activity itself.

More specifically, those undergoing BA treatment are encouraged to make structured attempts to increase engagement in positively reinforcing behaviors (e.g., pleasant events), while decreasing negatively reinforcing behaviors (e.g., avoidance) that could result in decreased wellbeing (Hopko, Lejuez, Ruggiero, \& Eifert, 2003). In its simplest sense, BA involves increasing pleasurable environmental events while decreasing aversive events. However, these are not the only possible additions and subtractions a client can make to his or her behavioral repertoire to 
improve well-being. Some clients may need to increase behaviors that will lead to more positive methods of coping, such as assertiveness or thought-stopping. Conversely, others may need to decrease certain actions that are associated with maladaptive coping, such as rumination. In BA, client thinking is acknowledged by the therapist, but more in the context of the consequences of the thinking behavior than in the actual content of thoughts (Martell, Addis, \& Jacobson, 2001).

Proponents of behavioral activation therapy emphasize the role of the behavioral consequences of inadequate coping within the shifting contexts of everyday life, while deemphasizing the role of biological and cognitive factors in the development of depression (Martell et al., 2001). Proper coping with the shifting contexts of daily life (e.g., moving, retirement, etc.) is a key determinant in staving off depression according to BA theory. Although advocates of BA acknowledge that biochemical factors (e.g., genes, neurotransmitters) as well as past experiences play a role in the development of depression, they feel that too much weight has been assigned to these factors as causes of depression, and assert that it is possible that the relation between mood and brain chemicals is bi-directional rather than unidirectional (Addis \& Martell, 2004). The BA model is viewed as cyclic, and takes into account the contribution of several different variables in the development of depression. Figure 1 depicts the BA model.

Behavioral activation has gained evidentiary momentum in the literature as a stand-alone treatment for depression, namely in the treatment of younger and middle-aged adults. Results of such studies are promising and continue to suggest that BA may be just as effective as the entire cognitive therapy program in the treatment of depression (Gortner, Gollan, Dobson, \& Jacobson, 1998; Jacobson et al., 1996) and also may be just as effective as CBT in preventing depression relapse at two-year follow-up (Gortner et al., 1998).

Dimidjian and colleagues (2006) conducted a randomized trial comparing behavioral 


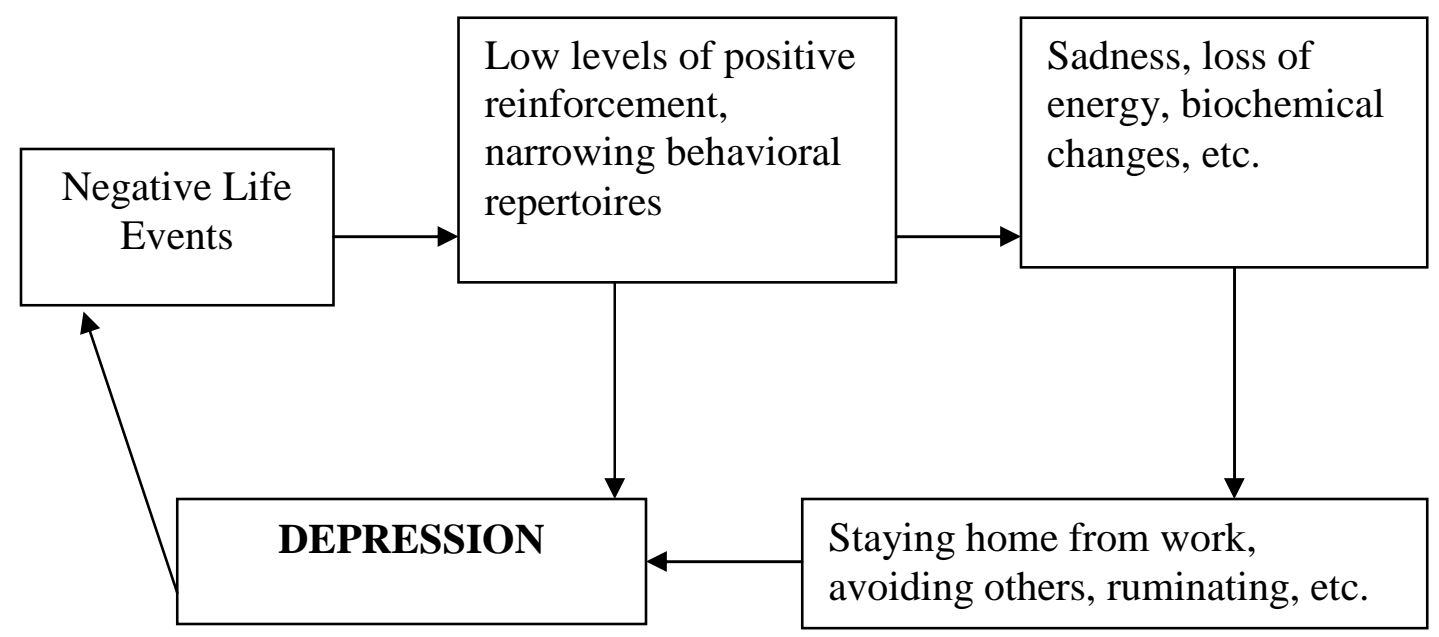

Figure 1. The BA model of depressed affect as presented by Martell et al., (2001).

activation, cognitive therapy, and antidepressant medication (Paroxetine). From this research, investigators found that BA was more efficacious than cognitive therapy and comparable in efficacy to antidepressant medication in the treatment of moderately to severely depressed adults following the acute treatment phase (Dimidjian et al., 2006). Compared to those in the antidepressant medication treatment condition, a significantly greater percentage of BA participants were both brought to remission and retained in treatment.

In a follow-up study, Dobson and colleagues (2008) examined the enduring effects of prior exposure to BA or cognitive therapy versus continued antidepressant treatment or pillplacebo withdrawal. Results demonstrated that both psychotherapies were at least as enduring (i.e., had comparable sustained depression recovery rates) as continued medication, and they were superior to pill-placebo withdrawal. Cognitive therapy conferred a slight advantage over BA in reducing relapse relative to pill-placebo withdrawal; however, rates of sustained recovery were not significantly different between the BA and cognitive therapy groups (Dobson et al., 2008). These findings are substantial, particularly because they suggest that psychotherapeutic methods are available that are just as efficacious as antidepressant medication (the current 
treatment standard). However, the cost of psychotherapy over time is much less (Dobson et al., 2008), and will not produce the side effects typically associated with pharmacotherapy.

Given that BA and cognitive therapies are relatively comparable in terms of symptom reduction and relapse risk, some may question the utility of developing and implementing the more contemporary treatment. One reason is that some have suggested that BA may serve as an alternate treatment in cases where cognitive therapy proves ineffective. For example, there was a subset of participants in the Dimidjian et al. (2006) study who provided a self-report of "extreme nonresponse" to cognitive therapy that was not exhibited on clinician ratings of depression; a pattern that was not observed in the BA group despite equivalent randomization to treatment condition (Coffman, Martell, Dimidjian, Gallop, \& Hollon, 2007). Coffman and colleagues (2007) have therefore suggested that perhaps some moderately to severely depressed individuals perceive better outcomes related to sustained attention to behavior change relative to a cognitive approach in the context of time-limited treatment. Along those lines, in a case study of a 62 yearold man with chronic treatment-resistant depression, researchers switched treatments after 147 days (21 sessions) of cognitive therapy to 147 days (14 sessions) of BA and found not only that BA significantly outperformed CT overall, but also that the rate of change in mood varied significantly between CT and BA (Bottonari, Roberts, Thomas, \& Read, 2008). Further examination of the participant's self-reported mood scores showed that mood steadily declined over the course of CT, whereas mood improved over the course of BA (Bottanari et al., 2008). Based on these findings, Bottanari and colleagues (2008) suggested that BA might be recommended in cases where there is concern that cognitive therapy may paradoxically trigger depressive rumination. Another reason to consider developing BA as a treatment involves its pragmatic approach and relative simplicity. Given BA may be more practical and easily 
understood than other treatments, it may be more easily learned and implemented by a wider scope of professionals and paraprofessionals, making it potentially a more affordable treatment option (Yon \& Scogin, 2009). The therapy's pragmatism and simplicity may also result in it being viewed as a more acceptable treatment approach by specific groups of potential therapy clients (e.g., those who are more action-oriented).

The availability of the Addis and Martell (2004) behavioral activation workbook provides individuals with depression or depressive symptoms even more options for receiving this promising treatment. This self-administered treatment is based on Martell et al.'s (2001) Depression in Context, which discusses BA theory and describes the treatment in a manualized, 'how-to' fashion. The workbook contains readings as well as built-in exercises to practice BA techniques (e.g., monitoring activity level, trying out new behaviors). For example, clients are taught to functionally assess their behavior and identify triggers that lead them to respond in a way that leads to negative personal consequences.

Often, this type of analysis leads to the identification of avoidance patterns that, although helpful in the short-term, frequently cause secondary problems for the individual (Martell et al., 2001). Those undergoing BA therapy are encouraged to identify behaviors that serve as inhibitors of well-being. The client is also asked to introduce or re-introduce pleasant behaviors into his or her behavioral repertoire that will aid in the improvement of mood by providing more positive reinforcement within the context of the client's environment. Then, taking everything into account, the individual develops treatment goals that will improve coping and ensure compliance with positively-reinforcing behaviors. The client is encouraged to "act towards a goal" in spite of internal mood state (Martell et al., 2001), under the expectation that one's mood will improve with increased activity level. (For more information on the history, methodology, or 
other issues related to face-to-face or self-administered behavioral activation therapy not mentioned here, please consult Addis \& Martell, 2004; Jacobson et al., 2001; or Martell et al., 2001).

Overcoming Depression One Step at a Time (Addis \& Martell, 2004) was included in the aforementioned recent rating study of the 50 top-selling self-help books for anxiety, depressive, and trauma-related disorders (Redding et al., 2008). Scores on the rating measure had a possible range of 19-95, with actual scores ranging from 34-94. Addis and Martell's (2004) behavioral activation workbook was given an overall rating score of 69 , ranking it in the $20^{\text {th }}$ position (Redding et al., 2008) and indicating that it is of relatively high quality. The BA workbook was outranked by six other self-help books for depression. However, the workbook appears to be ranked relatively highly given the paucity of psychological research available on BA to substantiate its merits. Unfortunately, specific data regarding the raters' perceived strengths and weaknesses of particular texts have not been published.

\section{Behavioral Activation with Older Adults}

To date, no articles have been published on behavioral activation bibliotherapy in any population, and few have been published regarding the face-to-face treatment's effectiveness in treating older adults with depression. In fact, it has only very recently become a topic of exploration. In a small $n$, multiple baseline pilot investigation of the effects of BA on older adults with depression, Yon and Scogin (2009) found that 71\% of participants no longer met criteria for a depressive disorder following a course of individual BA psychotherapy. Furthermore, approximately $56 \%$ of participants experienced clinically significant improvement in symptoms, and the same percentage had post-treatment scores within normal limits on a self-report measure of depression (Yon \& Scogin, 2009). Another small $n$, multiple baseline study of BA as a 
treatment for below-average quality of life in older adults with activity-limiting health problems found significant improvements in distress related to somatic complaints as well as nearsignificant positive changes in positive affect and pleasant events following treatment (Moss \& Scogin, 2006). Although other measures (e.g., depression, activity restriction, quality of life, etc.) in the aforementioned investigation were trending in the appropriate direction, they did not reach significance. Due to the uncontrolled nature and small sample sizes of these investigations, it is difficult to make any statements about the merits or shortcomings of BA treatment. A more thorough investigation involving a larger sample size as well as a controlled comparison group is warranted to investigate the effect of the treatment on geriatric depression.

Proponents of BA have suggested that it may be a preferable treatment for older adults given that the approach is more pragmatic and possibly less cognitively demanding than other forms of therapy (e.g., cognitive therapy; Hollon, 2000). Also, because older adults have more difficulty reframing and controlling their thoughts (Hertzog \& Hultsch, 2000), it is thought that BA may be a more effective treatment for this population than treatments using cognitive techniques.

It is also speculated that BA may be a successful intervention for older adults because it is one method of addressing the problem of activity restriction. The activity restriction model of depressed affect states that perceived activity restriction caused by a major life stressor is a powerful predictor of psychological adjustment (Williamson \& Shaffer, 2000). Health-related issues (e.g., health conditions, disability, etc.) have been the most researched life stressors in the activity restriction literature due to the established link between health problems and depression. However, health problems are not thought to cause depression. Instead, it is often the activity restriction incurred by illnesses and disability that leads to depressive symptoms. For older 
adults, who are more likely to be affected by health problems than individuals in other age groups (Areán, Uncapher, \& Satre, 1998), the activity restriction model is particularly applicable. Activity restriction has been found to mediate the association between medically-related stressors and depression, even after controlling for other known causes of depression among older adults (Williamson \& Shaffer, 2000). These findings have been replicated a number of times with a variety of activity-restricting health problems, from limb amputees (Williamson, Schulz, Bridges, \& Behan, 1994) to breast cancer patients (Williamson, 2000) to those who struggle with pain (Williamson \& Schulz, 1992).

In a recent study of older women with activity restriction due to osteoarthritis, it was found that higher perceived control and greater use of active coping strategies were related to lower depressive symptomatology, whereas increased use of avoidant coping strategies (particularly behavioral disengagement) were related to greater depressive symptomatology (Rivard, 2007). These findings map on well to BA, given the skills associated with staving off depression are skills that are stressed in the treatment. The notion that BA may be a successful intervention for older adults because it addresses the problem of activity restriction has not been empirically validated. However, it is likely that a treatment that focuses on active coping within the shifting contexts of life (including changes in health) would be well-suited to a group that is often affected by health-related problems.

\section{Need for Study}

Depression spectrum disorders and depressive symptoms are among the most commonly occurring psychological conditions in late life. Although some treatments have been identified as evidence-based in this population, researchers and practitioners alike continue to search for simpler and more cost-effective methods of treating depression. Behavioral activation therapy, a 
newer behavioral treatment that has been developed from the pure behavioral component of cognitive-behavioral therapy, is seen as advantageous over other approaches (e.g., cognitivebehavioral or insight therapies) in that it is pragmatic and may require less of both the therapist and client alike with comparable effects on outcome. BA has been established in controlled studies to be just as efficacious or superior to cognitive therapy and just as efficacious as antidepressant medication in the treatment of younger and middle-aged adults with depression. Further, smaller investigations looking at BA as a treatment for late-life depression and belowaverage quality of life have yielded promising results. A behavioral activation bibliotherapy workbook has been developed, but to date no studies have been conducted examining its efficacy in treating depression in any age bracket. Bibliotherapy treatments are known to apply well to the symptom presentations and practical needs of depressed older adults. Therefore, a study examining a behavioral activation bibliotherapy treatment for late-life depression would be a meaningful addition to the literature.

\section{Current study}

The present study investigated behavioral activation bibliotherapy as a treatment for latelife depressive symptoms. BA bibliotherapy was administered using Addis and Martell's (2004) Overcoming Depression One Step at a Time as a stand-alone treatment that was completed by participants over a 4-week period. Results of the immediate intervention group were compared with those of a delayed treatment control group and both groups' treatment response was followed one month after treatment completion. 


\section{Hypotheses}

Two main hypotheses were evaluated in this investigation:

1) It was hypothesized that participants in the intervention group would have significantly lower scores on the Hamilton Rating Scale for Depression (HRSD; Hamilton, 1967) following treatment than the control group.

2) It was also hypothesized that scores on the Geriatric Depression Scale (GDS; Yesavage et al., 1983) would be significantly lower for the intervention group than the control group following treatment.

\section{Research Questions}

Secondary to the main hypotheses, the following additional exploratory research questions were examined:

1) Would scores on the other dependent measures included in the study (i.e., positive affect on the Positive Affect subscale of the Positive and Negative Affect Schedule (PANAS; Watson, Clark, \& Tellegen, 1988) and pleasant events as reflected by responses on the Pleasant Events Scale-Elderly-20 (PES-E-20; Teri \& Lewinsohn, 1982) increase significantly more for the intervention group following treatment?

2) Would depressive symptoms as measured by the HRSD and GDS significantly decrease for both treatment groups combined from pre-treatment through 1-month follow-up?

3) Would any treatment gains observed be maintained from post-treatment to 1-month followup as reflected by scores on the HRSD and GDS? 


\section{CHAPTER 2}

\section{METHOD}

Participants

\section{Recruitment}

Community-dwelling participants were recruited from two separate sites: 1) Tuscaloosa, Alabama and 2) Jamestown, New York. Participants were recruited within a 60-mile radius of the first site. Recruitment from the second site was initially planned within a 60-mile radius as well. However, due to the limited availability of project personnel in this location to respond to a potential crisis situation (i.e., suicidality), recruitment was limited to a group of senior housing facilities owned and operated by the same company who have service coordinators available to respond to crisis calls. The housing at the second site consisted of 320 apartments in 8 separate equal-opportunity complexes that are located either within the city of Jamestown, New York or in Falconer, NY, which is located less than 5 miles from the city of Jamestown. These residences provide affordable housing for those ages 62 and above. The primary investigator has an established relationship with the Housing Coordinator and Service Coordinators associated with these facilities.

Participants were recruited via a number of methods, including personal referrals from health care professionals, newspaper advertisements, flyers, psychoeducational talks about geriatric depression and health at locations frequented by older adults (e.g., senior housing facilities). Also, referrals were received from similar research investigations in which individuals 
interested in participation did not meet inclusion criteria for those projects, but did meet inclusion criteria for the present study.

Sample Size

This study aimed to reach an overall power level of 0.80 using an alpha level of .05 and an effect size of $d=1.0$. A power analysis was conducted to determine the appropriate sample size for the study. Based on a power calculation chart in Cohen (1988), it was established that 26 participants were needed for the current study. The effect size chosen for this study was settled on as a compromise between the mean effect size of similar self-help treatment studies in the researcher's lab $(d=1.16)$ and the mean effect size that was reported in Gregory and colleagues' (2004) meta-analysis of 29 bibliotherapy studies for depression $(d=0.88)$. This large effect size indicates that the intervention group was expected to produce one standard deviation more change than the control group. It was determined that an effect size of this magnitude would ensure sufficient power, minimizing the likelihood of a Type II error (significant results exist between the groups but are not found). Power could have been increased by raising the sample size; however, the length of the recruitment phase for this investigation limited the feasibility of this option. Power could have also been increased by relaxing the alpha level (e.g., to .10). However, this would have increased the likelihood of committing a Type I error and erroneously finding significant results. Therefore, it was decided to maintain the alpha, power, and effect size values used in the original power analysis.

\section{Sample Characteristics}

Those deemed eligible to participate in this investigation: 1) were community-dwelling, 2) were 50 years of age or older, 3) received a score of 33 or greater on the modified Telephone Interview for Cognitive Status (TICS-M; Welsh, Breitner, \& Magruder-Habib, 1993) to rule out 
possible dementia, and 4) received a score of at least 5 on the GDS (to be inclusive of the widest range possible of depressive symptoms while still allowing room for potential improvement in symptomatology). Individuals who 1) were actively suicidal, 2) had not been stabilized on antidepressant or anxiolytic medication for at least 1 month, 3) reported a psychotic or substance use disorder, 4) were currently receiving psychotherapy, or 5) were unable to read (as assessed by questions pertaining to current reading habits, enjoyment of reading, and feelings about receiving treatment requiring the reading of a book) were excluded from participation in this study.

The final sample consisted of 26 participants with 13 participants randomized into each of the two treatment conditions. Please refer to Table 1 for a visual depiction of the sample characteristics. Individuals in the study ranged in age from 65-89 years; $M=77.5, S D=6.72$. The sample consisted of 20 females (77\%) and 6 males (23\%). Sixteen participants (62\%) were recruited from the New York site, and the remaining 10 (38\%) were recruited from the Alabama site. The majority of the sample identified as being non-Hispanic white (81\%), followed by African American (15\%), and Native American (4\%). Fifteen participants in the study were widowed (58\%), 6 were married (23\%), 4 were divorced (15\%), and 1 was separated (4\%). Educational attainment ranged from $10-17$ years, $M=13.77$ years, $S D=2.03$. Annual household income varied quite a bit, with $39 \%$ of the sample reporting incomes at or below $\$ 15,000,15 \%$ reporting between $\$ 15,001-\$ 30,000,15 \%$ reporting $\$ 30,001-\$ 70,000,12 \%$ reporting $\$ 70,001-\$ 110,000$, and $19 \%$ were unaware of or refused to report income. In terms of perceived income adequacy, $23 \%$ of participants rated their annual household income as 'slightly' or 'very' inadequate, $31 \%$ as 'adequate,' and $46 \%$ as 'mostly' or 'very' adequate. Regarding self-reported global health status, $46 \%$ rated their health as 'fair' or 'poor', $27 \%$ as 
'good,' and 27\% as 'very good' or 'excellent.' Participants exhibited scores ranging from 33 to 44 on the TICS-M $(M=35.23, S D=3.14)$, indicating probable absence of cognitive impairment. Range of baseline depressive symptoms as measured by the GDS was 2-23; $M=9.31, S D=5.62$. On the HRSD, depressive symptoms ranged from 4 to $22 ; M=11.62, S D=6.03$.

\section{Design}

This study employed both between- and within-subjects designs to address the hypotheses and research questions. A between-subjects design was used to examine the preliminary efficacy of the behavioral activation bibliotherapy intervention to improve depressive symptoms (Hypotheses 1 and 2). This design was also used to examine the impact of the intervention on participants' pleasant events and positive affect (Research Question 1). Treatment groups consisted of an immediate intervention and a delayed treatment control. This design was chosen to determine whether the immediate, self-administered intervention would yield improvements above and beyond those evidenced through the passage of time in the delayed treatment control group. As the use of a delayed treatment group resulted in the loss of

study control after the second time of measurement, a within-subjects (repeated measures) design was used to examine changes in depressive symptoms for both treatment groups combined across repeated assessment periods (Research Questions 2 and 3).

Participants deemed eligible to participate in the project completed a baseline assessment (Time 1, or T1) battery (which served as a pre-treatment assessment battery for the immediate group) and were randomized to treatment condition. Those in the intervention group immediately participated in the 4-week intervention following the T1 assessment, while those in the control group had a 4-week delay. Following completion of the 4-week intervention or delay period, participants were assessed again (Time 2, or T2), which served as a post-treatment assessment 
Table 1

Characteristics of the Sample $(N=26)$

Demographic

$n$

$\%$

Site

New York

16

61.5

Alabama

10

38.5

Sex

Female

20

76.9

Male

6

23.1

Race/Ethnicity

Non-Hispanic White

African American

21

80.8

Native American

$4 \quad 15.4$

Marital Status

Widowed

Married

Divorced

1

3.8

Separated

Annual Household Income

$\$ 5,001-\$ 15,000$

15

57.7

6

4

23.1

15.4

$1 \quad 3.8$

$\$ 15,001-\$ 30,000$

10

38.5

$\$ 30,001-\$ 70,000$

4

4

15.4

$\$ 70,001-\$ 110,000$

15.4

Unknown/Refused

$\begin{array}{ll}3 & 11.5 \\ 5 & 19.2\end{array}$

Income Adequacy

Very Inadequate

Slightly Inadequate

Adequate

Mostly Adequate

Very Adequate

2

4

7.7

15.4

30.8

$\begin{array}{ll}8 & 30.8 \\ 4 & 15.4\end{array}$

Global Health Status

Poor

2

7.7

Fair

10

38.5

Good

26.9

Very Good

7

Excellent

5

19.2

2

7.7

Note. Mean age $=77.5, S D=6.72$, $($ range $=65-89)$; Mean years of formal education $=13.77, S D=2.03$, (range $=10-17$ ); Mean score on the Telephone Interview for Cognitive Status $($ TICS-M) $=35.23, S D=3.14$, $($ range $=33-44)$; Mean score on the Hamilton Rating Scale for Depression $(H R S D)=11.62, S D=6.03$, $($ range $=4-22)$;

Mean score on the Geriatric Depression Scale $(\mathrm{GDS})=9.31, S D=5.62$, $($ range $=2-23)$. 
for individuals in the immediate treatment group and a pre-treatment assessment for those in the delayed treatment control group. After assessment T2, participants in the delayed treatment group started their 4-week intervention period. At Time 3 (T3), participants in the immediate treatment group were administered a 1-month follow-up assessment whereas participants in the delayed treatment control group were administered a post-treatment assessment. Time 4 (T4) was given to delayed treatment control participants only, serving as a 1-month follow-up assessment for this group. An abbreviated 5-item GDS (Hoyl et al., 1999) was administered weekly during the self-help treatment phase for both groups to monitor symptoms. Depressive symptoms were monitored during the 4-week delay phase for control group participants via two questions: 1) "Have you noticed any changes in your mood over the past week?" and 2) "Have you had any thoughts of harming yourself over the past week?" Please refer to Table 2, which displays the schedule of assessments. Please see Figure 2 to review the project design.

\section{Sociodemographic Information}

\section{Measures}

Sociodemographic information was collected using a researcher-developed questionnaire that included variables such as sex, race, age, income and income adequacy, self-rated global health status, existing medical and mental health conditions, mental health history, and current and previous treatment received for depression and other mental health disorders. Information gathered from this measure was used to describe the sample and also to assess whether the randomization process produced equivalent groups based on sociodemographic variables.

\section{Cognitive Status}

The Telephone Interview for Cognitive Status - Modified (TICS-M; Welsh et al., 1993) was used to screen participants' cognitive status in an effort to determine whether potential participants were likely to have the ability to participate in the intervention. This 21 -item 
Table 2

Schedule of Assessments

1-mo.

\begin{tabular}{lllll} 
Measure & Screening & Baseline & Pre-tx & Treatment Post-tx follow-up \\
\hline
\end{tabular}

Demographic

Questionnaire

TICS-M X

GDS-5

GDS

HRSD

PES-E-20

PA

BAKT

Treatment

Enactment

Treatment

Benefit

X
X

$\mathrm{X}$

$\mathrm{X}$

X $\quad X$

X

X

X $\quad X$

X $\quad X$

X $\quad X$

X $\quad X$

X $\quad X$

X

X

X

X

X $\quad X$

$\mathrm{X}$

Note. Immediate intervention group had 3 major assessments (baseline, post-treatment, and follow-up), whereas delayed control group had 4 major assessments (baseline, pre-treatment, post-treatment, and follow-up). TICS-M = Telephone Interview for Cognitive Status - Modified; GDS-5 = 5-item Geriatric Depression Scale; GDS = Geriatric Depression Scale; HRSD = Hamilton Rating Scale for Depression; PES-E-20 = Pleasant Events Scale-Elderly-20; PA = Positive Affect subscale of the Positive and Negative Affect Schedule; BAKT = Behavioral Activation Knowledge Test

measure examines four domains of cognitive functioning: 1) Orientation, 2) Memory (comprised of registration and recent and delayed memory), 3) Attention/Calculation, and 4) Language (comprised of semantic memory, comprehension, and repetition tasks). A cutoff score of 33 out of a possible 50 was used to exclude those with potential cognitive impairment in this study, as a 


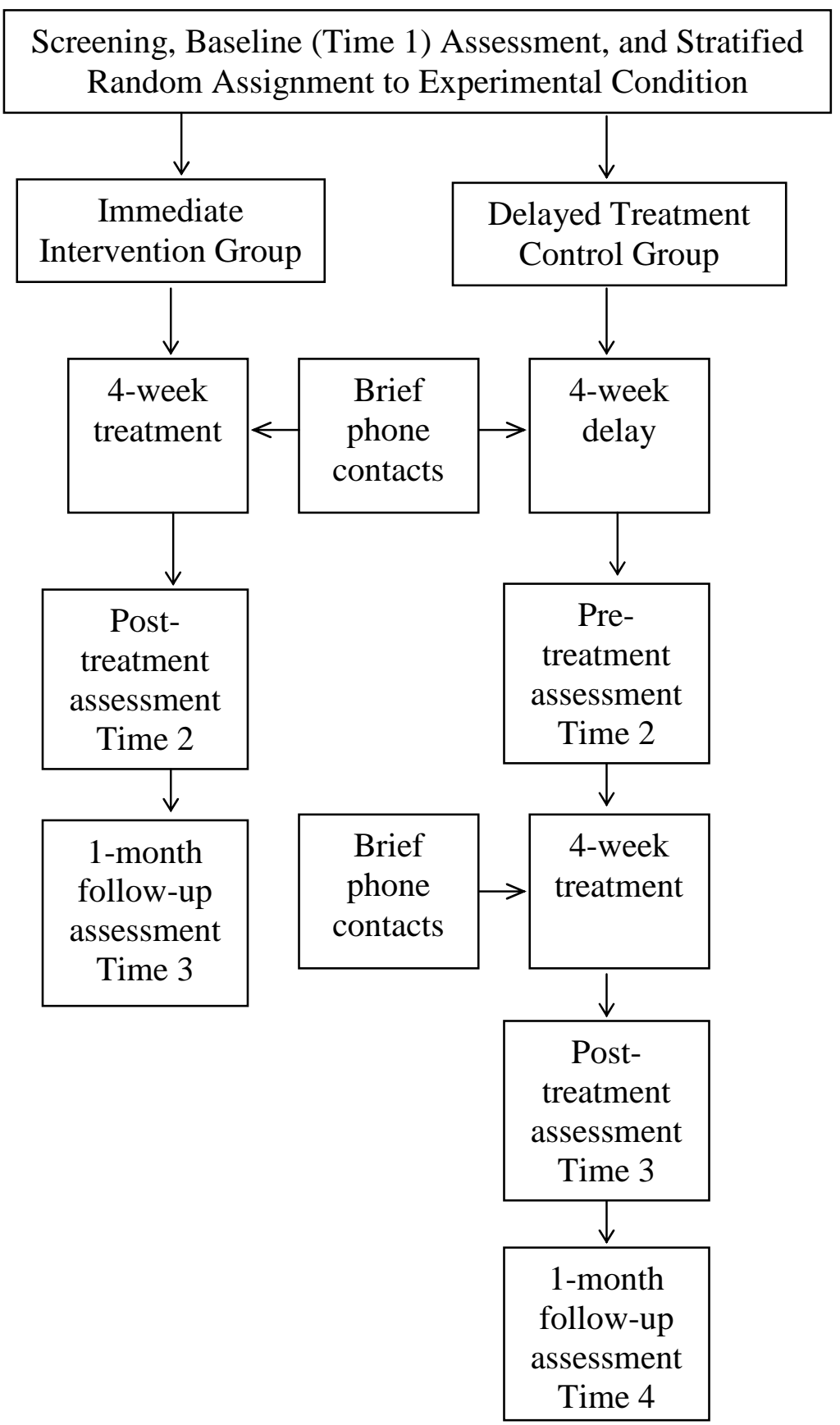

Figure 2. Design and procedures of the immediate intervention and delayed treatment groups.

score of 30 is typically the highest score associated with being at risk for developing dementia (Welsh et al., 1993). The TICS-M has good test-retest reliability as well as good concurrent 
validity with the MMSE, and is often preferred to the MMSE because it does not evidence a ceiling effect (e.g., Moylan et al., 2004).

\section{Depression}

Clinician-rated depression. The Hamilton Rating Scale for Depression (HRSD;

Hamilton, 1967) was used as the primary outcome measure. The HRSD is the clinician standard for assessing depression (Floyd, Scogin, \& Chaplin, 2004). This 17-item scale is administered in a semi-structured interview format and includes questions about various domains related to depression (e.g., depressed mood, insomnia, suicidality, etc.). Each item is rated on a domainspecific severity scale using a combination of participant self-report, participant observation, and clinical judgment. Scores on the measure range from $0-52$, with a score of 10 or above being indicative of significant depressive symptoms. For the purposes of this investigation, participants were asked to consider the past 2 weeks when responding to the questions.

Adequate reliability and validity has been established for this measure (Scogin, 1994). The HRSD has demonstrated concurrent validity of .72 with the Beck Depression Inventory (BDI; Beck, Ward, Mendelsohn, Mock, \& Erbaugh, 1961). It also has a test-retest reliability of .77 (Guy, 1976). In addition, the HRSD has demonstrated good concurrent validity with the GDS (Lyons, Strain, Hammer, \& Ackerman, 1989; Salamero \& Marcos, 1992). In the present study, there was a strong, positive correlation between the HRSD and GDS at baseline, $r=.74, p<$ .001 .

Due to the clinical skill required in rendering scores on this scale, graduate-level research staff members ( 2 individuals from the principal investigator (PI)'s research laboratory) were trained in the administration and scoring procedures by the PI and her research advisor. Raters, including the principal investigator, practiced scoring the measure on tape-recorded interviews 
from previous studies using an updated grid-based version of the HRSD (GRID-HAMD; Williams et al., 2008). This measure employs more structured, standardized procedures for administration and scoring that are thought to ensure higher reliability and validity than the original version of the HRSD (Williams et al., 2008). The GRID-HAMD contains the same items as the HRSD, but symptom frequency and intensity are separated out for the majority of items given these dimensions must be considered separately before an overall severity score can be assigned. The creators of the GRID-HAMD have also clarified some of the wording in the scoring portion of the HRSD and have added relevant clinical examples in an effort to make scoring less subjective. Inter-rater reliability on the GRID-HAMD was found to be high at 0.95 , and items were internally consistent $(\alpha=0.78)$, (Williams et al., 2009). In addition, Williams and colleagues (2009) reported that the measure has demonstrated good concurrent validity with the Structured Interview Guide for the Hamilton Depression Rating Scale (SIGH-D; Williams, 1988). For the present study, raters reached $94 \%$ agreement on HRSD scores prior to scoring any study-related measures. Inter-rater reliability was also assessed on a 10\% random sample of HRSD interviews gathered by graduate assistant raters on digital recording throughout the investigation. Raters reached $88 \%$ agreement on those assessments.

Self-reported depression. The Geriatric Depression Scale (GDS; Yesavage et al., 1983) is a 30-item measure designed specifically for use with older adults. Questions are presented in a dichotomous "yes/no" format (e.g., "Are you basically satisfied with your life?"). Internal consistency (Cronbach's alpha $=.94)$ and test-retest reliability $(r=.85)$ have been established for this measure (Scogin, Rohen \& Bailey, 2000). This instrument was used for both screening and outcome purposes. The traditional cutoff scores on this measure are as follows: $0-9=$ normal, $10-$ $19=\operatorname{mild} /$ moderate depression, $\geq 20=$ severe depression. However, a range of scores exists 
around the cutoff scores that are still inclusive of a particular severity level. For example, a score as low as 8 has been found to identify those with depression (Ertan, Ertan, Kiziltan, \& Uygucgil, 2005). Therefore, it is likely that an even lower score on this measure would screen for depressive symptoms. A screening cutoff value of 3 on the 15 -item version of the GDS has been used to identify participants with depressive symptoms (Snarski, 2007), which is equivalent to a score of 6 on the GDS. A screening cutoff score of 5 was used in this study to be inclusive of the broadest range of individuals possible across the depressive spectrum given that older adults often express subthreshold symptoms of the disorder (Blazer, 2002).

Although this measure was originally validated with individuals ages 65 and over, the GDS can be used with younger age groups as well. In their study of the psychometric properties of the GDS with younger populations, Rule, Harvey, and Dobbs (1989) found that the GDS is internally consistent (Cronbach's alpha $=.82)$ and has established convergent validity with the Zung Self Rating Depression Scale (Zung, 1965) in individuals ranging in age from 17-55 years old. In addition, in a second study noted in their 1989 article, the authors found that the GDS demonstrated good internal consistency (Cronbach's alpha $=.85$ ) among 389 individuals ages 30-65 (130 of which were ages 50-59). Based on these and other tests, the authors concluded that the GDS is sufficiently reliable for use with younger populations.

The GDS-5 (Hoyl et al., 1999) was used to monitor depressive symptoms on a weekly basis throughout the 4-week treatment phase of the study. This measure consists of 5 items from the GDS-15 (an abbreviated version of the original GDS). A score of 2 or greater is predictive of depression. When Hoyl and colleagues (1999) compared the GDS-5 with the GDS-15, it had a sensitivity of .97 , specificity of .85 , positive predictive value of .85 , negative predictive value of 
.97 , and accuracy of .90 for predicting depression. In addition, significant agreement was found between depression diagnosis and the GDS-5 (Hoyl et al., 1999).

Positive Affect

Although depression intervention research often focuses on decreasing negative affect, positive affect can increase secondarily as a result of treatment (e.g., Mohr et al., 2005; Moss \& Scogin, 2006). The 10-item Positive Affect subscale of the Positive and Negative Affect Schedule (PANAS; Watson, Clark, \& Tellegen, 1988) was used to evaluate positive affect over the course of the study. The Positive Affect (PA) scale describes the extent to which the client feels enthusiastic, active, alert, and engaged. The scale contains ten words, which are related to PA (e.g., "interested," "strong"). Clients are asked to indicate the extent to which each term pertains to them during a specified period of time (ranging from this moment or week to in general, depending on the study). Responses fall on a scale from 1 to 5 ( $1=$ very slightly or not at all, $2=$ a little, $3=$ moderately, $4=$ quite a bit, and $5=$ extremely). For this investigation, participants were asked to consider the past few weeks when generating item responses.

Test-retest reliability over an 8-week interval on the PA scale ranged from .47 to .68 and coefficient alphas ranged from .86 to .90 , depending on the time frame for which individuals responded (i.e., past few days vs. past year). The scale was found to be largely uncorrelated with the Negative Affect subscale (from -.23 to -.12). The PA scale was also found to demonstrate convergent and discriminant validity when correlated with various positive and negative affect mood scales. Psychometric properties have been conducted on this scale with older adult medical patients as well (Ostir, Smith, Smith, \& Ottenbacher, 2005). Test-retest reliability over a 90-day period was .79 and the coefficient alpha was .85 , demonstrating evidence that this scale can provide reliable and consistent information when used with older adults. 


\section{Pleasant Events}

Participants were administered the Older Adult Pleasant Events Scale-Elderly-20 (PESE-20) to rate their enjoyment $(0=$ A Great Deal, $1=$ Somewhat, or $2=$ Not at All $)$ and frequency of engagement in the past month $(0=$ Not at All, $1=1$ to 6 times, or $2=7$ or more times $)$ in a variety of activities such as "Being with someone I love" or "Spending time with animals." The PES-E-20 is an abbreviated version of the Pleasant Events Scale-Elderly (PES-E), a 114-item measure that was designed for the purpose of identifying pleasant events relevant to individuals over the age of 50 (Teri \& Lewinsohn, 1982). Because this measure is lengthy, the 20-item version was created and has been used in several research projects with older adults (e.g., Quality of Life and Older Rural Health Care Recipients, 1998; Yon \& Scogin, 2009). The scale is scored by summing the cross-products of enjoyment and frequency for each item. Higher scores indicate participation in more enjoyable activities. A coefficient of 0.81 was obtained on this measure, providing evidence of reliability (A. Yon, personal communication, September 10, 2007). Further, across a 3-month period, with a sample size of 35, a test-retest reliability of 0.42 was obtained indicating that there is adequate evidence for test-retest reliability over this interval (A. Yon, personal communication, September 10, 2007). As BA encourages clients to increase engagement in positively reinforcing activities, the PES-E-20 was used to measure engagement in and enjoyment of various activities known to be pleasant to those ages 50 and above.

\section{Treatment Implementation}

Treatment implementation involves the measurement of individual treatment components (delivery, receipt, and enactment) to ensure that a treatment is conducted as intended (Lichstein, Riedel, \& Grieve, 1994). 
Delivery. Treatment delivery, or the interventionist's ability to present the intervention to the participant as intended, was not assessed in this investigation because the treatment is selfadministered.

Receipt. Treatment receipt (the degree to which the participant actually received the intended treatment) was measured using an updated version of the Behavioral Activation Knowledge Test (BAKT; Moss, 2007). The original BAKT is a 29-item true/false format knowledge test that taps into the understanding of various aspects of BA. Some preliminary reliability analyses were conducted on the original version of this unpublished test with young adults and good internal consistency was found (Cronbach's alpha $=.89$ ). The updated version of this test was created for this investigation to modify questions on the original measure that were not applicable to this study (i.e., pertained only to individual, face-to-face BA treatment). In addition to modifications of individual items, two items were dropped from the original 29 for similar reasons. This measure was administered at both pre- and post-treatment to ascertain whether knowledge about BA would increase significantly over the course of the selfadministered program. The psychometric properties of the updated instrument have not been fully evaluated. However, an examination of the measure after the present study using the KuderRichardson Formula 20 (KR-20) yielded a value of .26, indicating that the internal consistency of this measure was poor.

Enactment. Treatment enactment (or the degree to which participants demonstrate changes in behavior in their natural environment related to the intervention) was assessed both briefly throughout treatment as well as more thoroughly post-treatment. On a weekly basis throughout the treatment phase, research staff recorded the percentage of pages read and written exercises completed as reported by the participant via phone. The amount of reading and 
exercises to be completed each week was suggested prior to the onset of treatment by the researcher through the provision of daily checklists. Therefore, the weekly percentages gathered via phone were based on the amount of suggested material to be completed. Following treatment, a similar measure examining percentage of pages read and written exercises accomplished was completed by research staff. Staff relied on self-report to ascertain the percentage of pages read at post-treatment. However, a more objective measure of written exercises completed was obtained by a physical count of the number of photocopied written exercises completed in a binder provided by staff prior to treatment. Please refer to the Procedure section of this document for more information regarding treatment enactment data collection.

\section{Treatment Benefit}

Following the treatment, participants were asked seven brief questions about the helpfulness and applicability of the treatment. Examples of such questions include, "On a scale from $0-10(0=$ least helpful; $10=$ most helpful $)$, rate how helpful the treatment was to you in general" or "On a scale from 0-10 ( $0=$ not well suited; $10=$ very well suited $)$, rate how well suited you feel behavioral activation treatment is to people your age."

\section{Procedure}

\section{Screening, Randomization, and Stratification}

Individuals interested in participation were provided a description of the study and asked for verbal assent to be screened via telephone (or in person if telephone screening was not feasible) using the GDS and TICS-M. Participants were also asked questions pertaining to their ability to read, frequency and enjoyment of reading, and whether or not they were comfortable and willing to be involved in a bibliotherapy intervention. 
Those eligible and with continued interest in participation were scheduled within the week to complete a baseline assessment battery (T1). These measures were administered in the client's home or over the telephone, depending upon participant preference. In instances where no preference was expressed, telephone was chosen as the primary method of assessment for both participant and researcher convenience. In-home baseline assessments were conducted with five participants; the remainder was completed via telephone. Prior to being administered the battery, participants provided written consent to treatment and research (during which time they were informed that they had a random chance to be entered into a delayed treatment group). Participants who were administered in-home assessments were given the consent form at the onset of the visit to review and sign. For those who were administered the baseline assessment over the phone, consent forms were mailed out to participants and sent back in a postage prepaid envelope prior to the telephone assessment session. The measures administered at baseline included the sociodemographic form, HRSD, GDS, PA scale, PES-E-20, and BA Knowledge Test. These assessments, with the exception of the sociodemographic form which was always given first, were counterbalanced to prevent any confounds caused by the order in which assessments were presented. This battery took no longer than 1 hour to complete in most cases. Following baseline assessment procedures, participants were randomized to either the immediate intervention or delayed treatment control condition. However, they were first stratified by site (Alabama (AL) and New York (NY)) and age ('young-old' (50-64) and 'olderold' (65+)). This procedure was used to ensure that a comparable proportion of individuals from each age/site combination were represented in both experimental conditions. It was also used to ensure that any extraneous factors present in the study were evenly distributed throughout treatment conditions, thus reducing threats to internal consistency. It should be noted that, 
because no 'young-old' adults were enrolled into the study as expected, randomization was stratified by site only. Please refer to Figure 3 for a depiction of the recruitment and stratification procedures for this study.

It is important to note that the stratification procedures did not ensure that equal numbers of individuals from each site and age group were included. Instead, when an individual from a particular site/age range combination entered into the study, he or she was randomly assigned to the intervention or control group. For this investigation, four lists were generated, each representing a stratum of the proposed study population (List $1=\mathrm{NY}$, 'young-old'; List $2=\mathrm{NY}$, 'older-old'; List 3 = AL, 'young-old'; List 4 = AL, 'older-old'). However, because no individuals under the age of 65 were enrolled in the study, only Lists 2 and 4 were used. Each list contained a set of the numbers 1 (meaning experimental) and 2 (meaning control) in random order. Once a participant was identified by age group and site, these lists were used by research staff to randomly assign that individual to the experimental or control group. Each of the individuals in each of the age/site combinations had an equal opportunity to be randomized into the experimental and control groups. Please refer to Figure 3 to review the number of participants in each stratification cell.

In this investigation, obtaining an equal number of individuals from the two age/site groups was not considered essential because the outcomes of Alabamians versus New Yorkers were not compared in this study. The aim of this project was simply to see if the program would exhibit preliminary efficacy in treating depressive symptoms in the overall study sample.

Although the empirical questions of differences in treatment outcome as a function of age and site are undoubtedly interesting, the ability of a study of this nature to detect any differences based on those variables is limited given the sample size and lack of control of the participant 


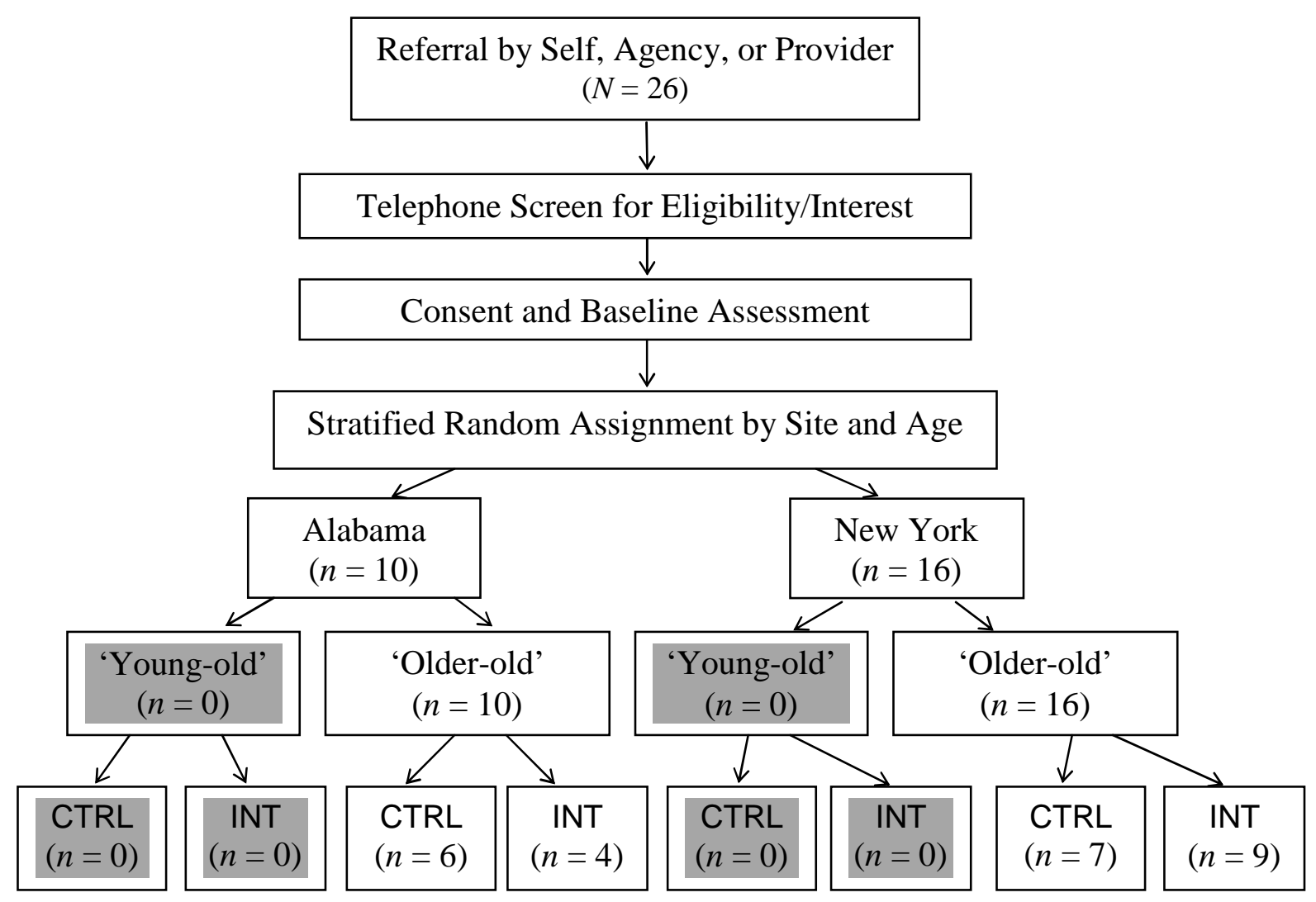

Figure 3. The study enrollment process with respect to stratification. Note that 'Young-old' stratification cells are shaded, as no younger-old adults were enrolled in the study as expected. $\mathrm{CTRL}=$ Delayed treatment control group; INT $=$ Immediate intervention group

numbers in each of the age/site groups (who self-selected into the project).

\section{Intervention}

Immediate group. Following completion of the baseline assessment battery and randomization to experimental condition, participants entered into treatment. Participants were provided Addis and Martell's (2004) Overcoming Depression One Step at a Time. This workbook is a 180-page presentation of behavioral activation bibliotherapy for depression. Following procedures by Flesch (1951), it was determined that the text falls at an estimated eighth-grade reading level. Examples of chapters in the book are "How Depression Works," "Learn Your Patterns and Start to Change Them," and "Free Yourself from Mood Dependence." The workbook contains 112 pages of treatment-oriented text, with the remainder of pages 
consisting of written exercises and an appendix of blank written exercise forms. Examples of written exercises in the book include completing activity-and-mood monitoring charts, identifying avoidant behaviors that contribute to depression, and identifying healthy alternative coping behaviors. In addition to the workbook, a binder was provided by the researcher which included introductory information pages briefly describing the text and BA treatment, guidelines for the amount of material to be completed each week, and photocopied written exercises from the book. Individuals in the study were instructed to read the entire workbook and complete the written exercises in the binder provided over a 4-week period following the suggested guidelines. Although self-administered treatments are typically designed to be self-paced, participants in this investigation were encouraged to adhere to a 4-week reading period due to the timeframe available for the completion of the study.

During the 4-week intervention period, the PI, who was supervised by a licensed clinical psychologist, contacted those undergoing treatment via phone on a weekly basis. The purpose of these brief, 5-10 minute phone contacts was to check for any general questions about the study, monitor depressive symptoms using the GDS-5, ensure that participants were not experiencing any notable deterioration in symptoms, and check progress on the readings and written assignments against the guidelines set forth by the researcher. No psychotherapy was provided during these phone calls. A protocol was developed to address the potential circumstance of a participant expressing immediate danger to self or others. However, the use of this protocol was not needed during the investigation.

Following the 4-week intervention period, participants were scheduled for a posttreatment assessment, which consisted of the HRSD, GDS, PA scale, PES-E-20, BAKT, and Treatment Benefit measures. This assessment session was also to be conducted in-home or over 
the phone depending on participant preference, with the default method of assessment being telephone when no preference was expressed. All of these assessments were completed via telephone. All efforts were made to blind assessors to experimental condition. However, participants were not instructed to refrain from revealing their experimental condition to interviewers, introducing a potential confound into the investigation. Measures at this assessment period were also counterbalanced. At the end of the assessment session, participants were reminded that they would be contacted in approximately one month to schedule a one-month follow-up assessment. After the post-treatment assessment session, participants were sent a postage pre-paid envelope in which to return the binders that were provided at the onset of treatment so that treatment enactment information could be obtained. Participants were permitted to keep their workbooks. They were also mailed $\$ 10$ in exchange for their participation in the post-treatment assessment. However, in many cases, participants expressed a preference to receive the total $\$ 20$ participation incentive following the one-month follow-up assessment in lieu of receiving $\$ 10$ at post-treatment and $\$ 10$ at one-month follow-up.

Finally, at one-month follow-up, participants were again administered the HRSD, GDS, PA scale, and PES-E-20 to monitor changes in symptoms. All of these assessments were completed over the telephone. Again, measures were counterbalanced to prevent any confounds caused by order effects. Upon completion of this assessment, participants were mailed either the $\$ 10$ one-month follow-up incentive or the full \$20 participation incentive, depending on the individual preference that was expressed at the post-treatment assessment.

Control group. Following completion of the baseline assessment battery, persons in the control group were informed that they would be receiving bibliotherapy treatment after a 4-week delay. Those in this group received weekly phone calls from the PI lasting approximately 5-10 
minutes in duration over this waiting period to thank them for their participation, remind them of the upcoming pre-treatment assessment, monitor distress, and prevent dropout. These calls were also completed to ensure comparable procedures among the two treatment groups (other than the self-administered treatment) in an effort to limit threats to internal validity. Following the 4-week delay, control group participants were scheduled for a pre-treatment assessment battery, which consisted of the same measures administered at the baseline assessment. Again, all efforts were made to blind assessors to experimental condition. Treatment commenced as soon as possible following the pre-treatment assessment and the exact procedures that were described for the immediate intervention group were carried out, including the one-month follow-up assessment. 


\section{CHAPTER 3}

\section{RESULTS}

\section{Equivalent Randomization}

An alpha level of .05 was used for all comparisons made in this investigation. An assessment of equivalent randomization to treatment condition was first conducted to ensure that the randomization process produced groups that were comparable. Continuous variables (i.e., age, education, cognitive ability, reading frequency and enjoyment, and pre-treatment scores on the HRSD, GDS, PES-E-20, PA, and BAKT) were evaluated using a series of independent samples $t$-tests. No significant differences were found between treatment groups on any of these variables. A Chi-square test for independence was first considered to examine categorical variables (i.e., sex, site, race, marital status, self-rated global health status, and presence of reading concerns). However, this test requires that the lowest expected frequency in any given comparison cell be at least 10 when investigating variables with 2 categories, and at least 5 when examining additional categories (Pallant, 2001). Due to the sample size of the present study, this criterion was not met for all but one of the variables (site). Therefore, Fisher's Exact Probability Test was used instead to compare categorical variables with two categories (i.e., sex, site, study completion, and presence of reading concerns) because it does not require a lower bound in terms of cell frequency. No significant differences were found between treatment groups on any of these variables. For categorical variables with three or more levels (i.e., race, marital status, and self-rated global health status), cell frequencies were examined visually to determine if any of these characteristics varied to a degree that might be considered problematic in terms of group 
equivalence. Overall, these analyses suggested that the randomization procedure produced groups that were relatively equivalent.

\section{Attrition}

Of the full sample of 26 participants, $69 \%(n=18)$ completed the full treatment program (i.e., through 1-month follow-up [T4]). This attrition rate is comparable to similar bibliotherapy investigations (e.g., Mckendree-Smith, 2000; Scogin, Hamblin, \& Beutler, 1987). The remaining eight participants dropped out of the program between the baseline and post-treatment assessments. Six of the eight participants discontinued participation due to health-related issues: Two individuals were diagnosed with cancer, one learned he/she had to have surgery for a preexisting (i.e., had prior to the study) health condition, one was hospitalized for worsening of a pre-existing health condition, and two perceived exacerbations of pre-existing health conditions (unrelated to study participation) that they felt precluded their ability to fully participate in the investigation. Another participant dropped out of the study because the individual felt the readings were not applicable to his/her situation after reading the introductory pages of the selfhelp book. The remaining participant discontinued participation because the individual had to move a significant distance away from one of the sites unexpectedly.

In cases in which data were missing for statistical analyses due to participant attrition, the last data points collected on the various measures were carried forward and entered as the subsequent data points. This effort was undertaken to ensure inclusion of all possible cases in the data analyses.

A series of independent-sample $t$-tests was conducted to test whether any differences existed between completers and non-completers on various continuous sociodemographic and pre-treatment variables. These variables included participant age, educational attainment, 
cognitive ability, reading frequency and enjoyment, and baseline depression scores on both the HRSD and GDS. Of these comparisons, reading enjoyment was the only variable found to be significantly different between the groups, $t(23)=-3.40, p=.002$, such that mean self-rated enjoyment of reading in those who discontinued participation was significantly lower than that of those who completed the program. Fisher's Exact Probability Test was used to compare categorical variables with two categories (i.e., group assignment, sex, site, and reading concerns). No significant differences were found between treatment completers and noncompleters on these variables. Cell frequencies were examined visually in categorical variables with three or more levels (i.e., race and self-rated global health status). These results showed that $100 \%$ of those who dropped out of the program $(n=8)$ had rated their health as either 'fair' or 'good' at baseline, compared to $50 \%(n=9)$ in those who completed the study. Of the remaining completers, 39\% $(n=7)$ rated their health as 'very good' or 'excellent,' and $11 \%(n=2)$ rated their health as 'poor.' Further, $100 \%$ of African American participants $(n=4), 67 \%$ of nonHispanic white participants $(n=14)$, and $0 \%$ of Native American participants $(n=1)$ completed the program. Although the findings related to race were different between completers and noncompleters when examined visually, little can be interpreted about these results due to the nature of the representation of racial/ethnic groups enrolled in the study. The findings related to health and reading enjoyment could hypothetically have had an effect on treatment outcome. However, there were no significant differences between treatment groups on these variables. This would suggest that any potential error introduced into the study by differences between completers and non-completers was equally distributed over the treatment conditions. Therefore, it is thought that attrition likely did not have a differential effect on treatment condition and related outcomes. Consequently, these differences were not statistically controlled for in the following analyses. 


\section{Main Hypotheses}

It was hypothesized that participants in the intervention group would have significantly lower scores on both the Hamilton Rating Scale for Depression (HRSD; Hamilton, 1967) and the Geriatric Depression Scale (GDS; Yesavage et al., 1983) following treatment than the control group. One-way between-groups analyses of covariance (ANCOVAs) were conducted to compare depression scores on the HRSD and GDS between the immediate intervention and delayed treatment control groups at assessment T2 (i.e., following treatment for the immediate group and following delay for the control group). Preliminary checks were conducted to ensure that there was no violation of the assumptions of normality, linearity, homogeneity of variances, homogeneity of regression slopes, and reliable measurement of the covariates.

In the HRSD analysis, the independent variable was the treatment group and the dependent variable consisted of HRSD scores at T2. Participants' scores on the baseline (T1) administration of the HRSD were used as the covariate in this analysis. After adjusting for preintervention scores, there was a significant difference between the two treatment groups on T2 HRSD scores, $F(1,23)=10.16, p=.004$, partial eta squared $=.31$. The T1 HRSD covariate explained $38 \%$ of the variance in T2 HRSD scores, partial eta squared $=.38$. Post-hoc visual comparison of the raw means suggested that depressive symptoms on the HRSD were significantly lower at T2 for the immediate treatment group $(M=5.46, S D=4.96)$ than they were for the delayed treatment control group $(M=10.85, S D=4.96)$. The effect size of this difference was large; $d=1.08$.

In the GDS analysis, the independent variable was the treatment group and the dependent variable consisted of GDS scores administered at T2. Participants' scores on the baseline (T1) administration of the GDS were used as the covariate in this analysis. After adjusting for pre- 
intervention scores, there was not a significant difference between the two treatment groups on T2 GDS scores, $F(1,23)=.02, p=.89$, partial eta squared $=.001$. Baseline (T1) GDS scores explained $43 \%$ of the variance in T2 GDS scores, partial eta squared $=.43$. Please refer to Figure 4 for a graphical display of the results of the main hypotheses.

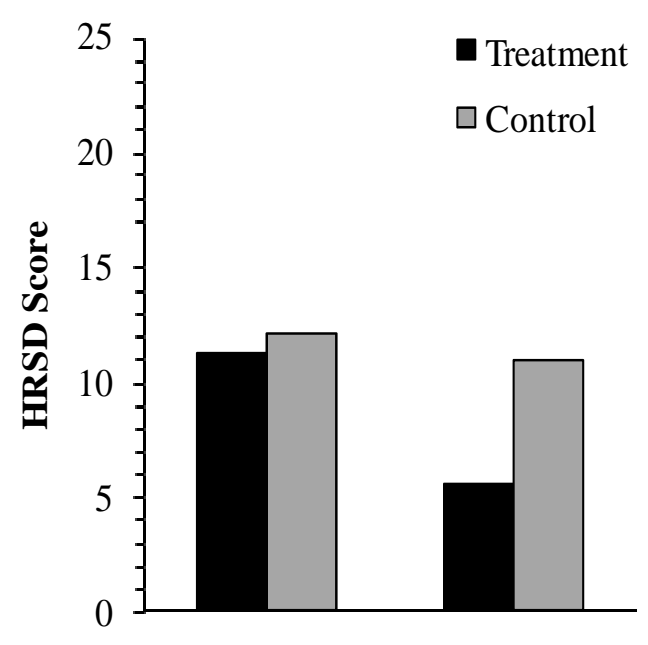

Time $1 \quad$ Time 2

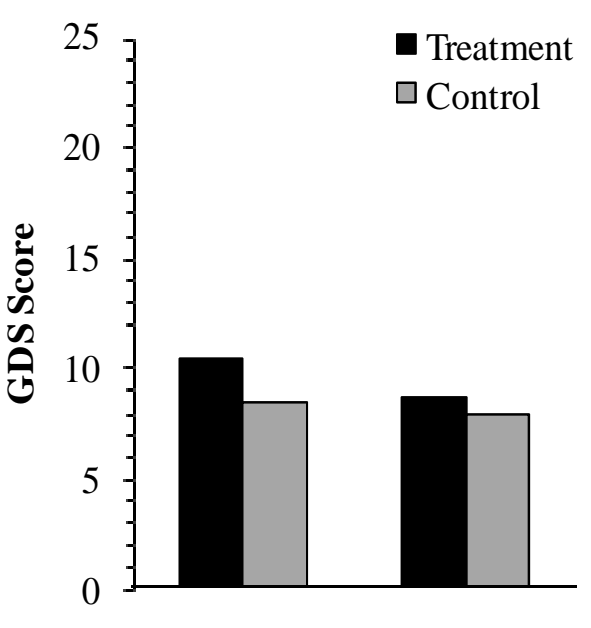

Time $1 \quad$ Time 2

Figure 4. Depressive symptoms at T1 and T2. Experimental groups differed significantly at T2 on the HRSD, but not on the GDS.

\section{Research Questions}

Research Question 1: Between-groups analyses. One-way between-groups analyses of covariance (ANCOVAs) were also conducted to compare scores on both the PA and PES-E-20 measures between the immediate intervention and delayed treatment control groups at T2. Preliminary checks of assumption violations were conducted for these analyses as well. In the PA analysis, the independent variable was the treatment group and the dependent variable consisted of scores on the Positive Affect scale at T2. Participants' scores on the baseline (T1) administration of the PA scale were used as the covariate in this analysis. After adjusting for preintervention scores, there was not a significant difference between the two treatment groups on 
T2 PA scores, $F(1,23)=1.89, p=.18$, partial eta squared $=.08$. The T1 PA covariate explained $26 \%$ of the variance in T2 PA scores, partial eta squared $=.26$.

In the PES-E-20 analysis, the independent variable again was treatment group assignment and the dependent variable consisted of PES-E-20 scores at T2. Participants' scores on the baseline (T1) administration of the PES-E-20 were used as the covariate in this analysis. After adjusting for pre-intervention scores, there was not a significant difference between the two treatment groups on T2 PES-E-20 scores, $F(1,23)=.72, p=.41$, partial eta squared $=.03$. Baseline (T1) PES-E-20 scores explained 32\% of the variance in T2 PES-E-20 scores, partial eta squared $=.32$. Please refer to Table 3 to review the mean scores for the variables used for the ANCOVAs by treatment group and Table 4 to review ANCOVA results.

Research Questions 2 and 3: Within-subjects analyses. Treatment outcome scores for both treatment conditions were combined for these analyses. One-way repeated measures ANOVAs were used to compare scores on both the HRSD and GDS at pre-treatment, post-treatment, and 1-month follow-up. There was a significant main effect for time on the HRSD, $F(2,24)=11.94$, $p<.001$, multivariate partial eta squared $=.50$. Post-hoc comparisons using Fisher's LSD suggested that depressive symptoms on the HRSD were rated significantly lower at both posttreatment $(M=5.96, S D=6.20, p<.001)$ and 1-month follow-up $(M=6.96, S D=6.47, p<$ $.001)$ than they were at pre-treatment $(M=11.04, S D=5.71)$. Effect sizes for these differences were large $(d=.84)$ and medium $(d=0.66)$, respectively. However, scores on the HRSD were rated significantly higher at 1 -month follow-up than they were at post-treatment, $p=.05$. A significant main effect for time was also found on the $\operatorname{GDS}, F(2,24)=4.94, p=.02$, multivariate partial eta squared $=.29$. Post-hoc comparisons using Fisher's LSD suggested that depressive symptoms on the GDS were not rated significantly lower at post-treatment $(M=8.00, S D=4.87)$ 
Table 3

Raw Means of Dependent Variables and Covariates by Group for ANCOVA Analyses

$$
\underline{\text { Immediate Treatment Group }(n=13) \quad \text { Delayed Treatment Group }(n=13)}
$$

\begin{tabular}{lllllllll} 
& \multicolumn{2}{c}{ Time 1 } & \multicolumn{2}{c}{ Time 2 } & \multicolumn{2}{c}{ Time 1 } & \multicolumn{2}{c}{ Time 2 } \\
Variable & $M$ & $S D$ & $M$ & $S D$ & $M$ & $S D$ & $M$ & $S D$ \\
\hline HRSD & 11.23 & 6.57 & 5.46 & 4.96 & 12.00 & 5.67 & 10.85 & 4.96 \\
GDS & 10.31 & 6.75 & 8.62 & 4.98 & 8.31 & 4.25 & 7.77 & 4.00 \\
PES-E-20 & 48.38 & 10.99 & 55.23 & 9.04 & 56.31 & 14.50 & 55.77 & 11.84 \\
PA & 30.46 & 7.04 & 33.85 & 6.93 & 34.38 & 7.59 & 32.38 & 6.54 \\
\hline
\end{tabular}

Note. HRSD = Hamilton Rating Scale for Depression; GDS = Geriatric Depression Scale; PESE-20 = Pleasant Events Scale-Elderly-20; PA = Positive Affect Scale of the Positive and Negative Affect Schedule.

than they were at pre-treatment $(M=9.04, S D=5.59), p=.22$. However, scores on the GDS were significantly lower at 1 -month follow-up $(M=7.23, S D=5.11)$ than they were at pretreatment, $p=.02$. The effect size of this difference was small, $d=.33$. Although scores on the GDS were lower at 1-month follow-up than they were at post-treatment, this result was not significant, $p=.08$. Please refer to Table 5 to review the mean scores used for the within-subjects analyses on the HRSD and GDS and Figure 5 for a graphical representation of these scores.

\section{Clinical Significance}

Clinically significant change on the HRSD and GDS was assessed following procedures suggested by Jacobson and Truax (1991). For an outcome score to reach clinical significance, two criteria are typically examined. First, a cutoff score is determined indicating that participants' scores following treatment are closer to that of the normally functioning population than they were prior to treatment. This criterion of clinical significance was not assessed for this study given mean depression scores at baseline on the GDS fell within normal limits and mean scores on the HRSD at baseline were within one point of those of the normally functioning 
Table 4

Between-Group ANCOVAs Examining Outcome Measures at T2 Controlling for T1

\begin{tabular}{|c|c|c|c|c|}
\hline Source & $d f$ & $F$ & $\eta_{\mathrm{p}}^{2}$ & $p$ \\
\hline \multicolumn{5}{|l|}{$\overline{\text { HRSD }}$} \\
\hline Group & 1 & 10.16 & .31 & .004 \\
\hline HRSD at TI & 1 & 14.10 & .38 & .001 \\
\hline Error & 23 & $(15.93)$ & & \\
\hline \multicolumn{5}{|l|}{ GDS } \\
\hline Group & 1 & .02 & .001 & .89 \\
\hline GDS at $\mathrm{T} 1$ & 1 & 17.29 & .43 & $<.001$ \\
\hline Error & 23 & $(12.15)$ & & \\
\hline \multicolumn{5}{|l|}{ PES-E-20 } \\
\hline Group & 1 & .72 & .03 & .41 \\
\hline PES-E-20 at T1 & 1 & 10.57 & .32 & .004 \\
\hline Error & 23 & $(79.32)$ & & \\
\hline \multicolumn{5}{|l|}{ PA } \\
\hline Group & 1 & 1.89 & .08 & .18 \\
\hline PA at T1 & 1 & 8.14 & .26 & .01 \\
\hline Error & 23 & $(34.97)$ & & \\
\hline
\end{tabular}

Note. Values in parentheses represent mean square errors. HRSD = Hamilton Rating Scale for Depression; GDS = Geriatric Depression Scale; PES-E-20 = Pleasant Events Scale-Elderly-20; $\mathrm{PA}=$ Positive Affect Scale of the Positive and Negative Affect Schedule.

Table 5

Pre-treatment, Post-treatment, and 1-month Follow-up Means for Within-Subjects ANOVAs

\begin{tabular}{lcccccc} 
& \multicolumn{2}{c}{ Pre-treatment } & \multicolumn{2}{c}{ Post-treatment } & \multicolumn{2}{c}{ 1-month follow-up } \\
Variable & $\mathrm{M}$ & SD & $\mathrm{M}$ & SD & M & SD \\
\hline \multirow{3}{*}{ HRSD } & 11.04 & 5.71 & 5.96 & 6.20 & 6.96 & 6.47 \\
GDS & 9.04 & 5.59 & 8.00 & 4.87 & 7.23 & 5.11 \\
& & & & & & \\
\hline
\end{tabular}

Note HRSD = Hamilton Rating Scale for Depression; GDS = Geriatric Depression Scale. 


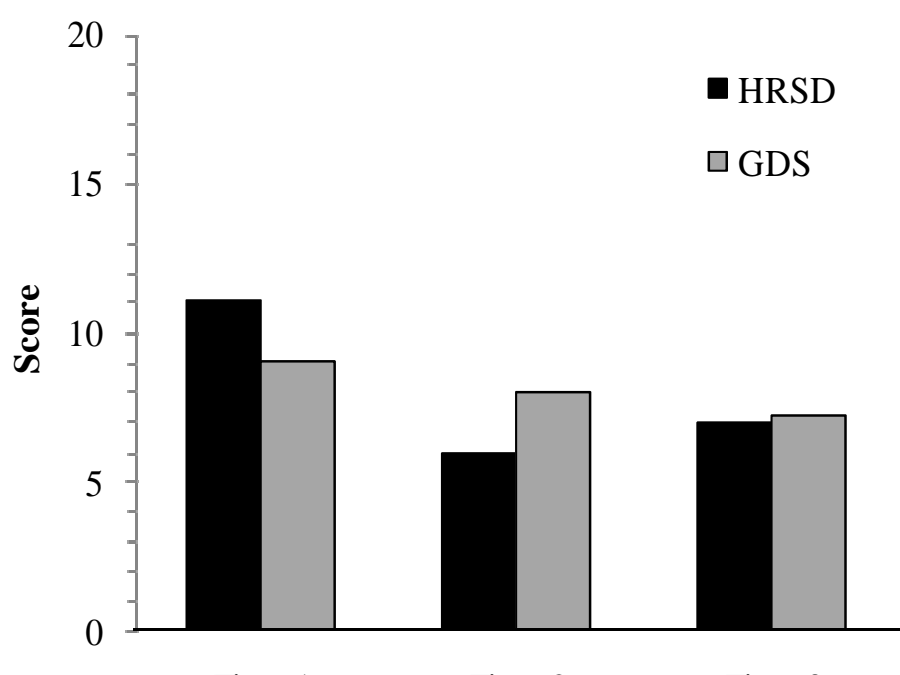

Time 1

Time 2

Time 3

Figure 5. Significant within-subjects main effects for Time on both the HRSD and GDS. T1 = pre-treatment, T2 = post-treatment, and T3 $=1$-month follow-up for these analyses. HRSD T1 to T2 and T1 to T3 significantly lower, $p<.001$. HRSD T2 to T3 significantly higher, $p=.05$. GDS T1 to T3 significantly lower, $p=.02$. All other findings did not reach significance.

population.

Second, a reliable change index $(\mathrm{RCI})$ is calculated to determine whether the magnitude of change for each participant is statistically reliable (i.e., change evidenced was due to more than random error in measurement alone). An index score of 1.96 is indicative of reliable change. RCIs were calculated from pre- to post-treatment for this investigation on individuals who completed the project. Those whose data were carried forward were excluded from these analyses. Of the 18 participants who completed the study, 39\% $(n=7)$ showed reliable change on the HRSD, whereas only $6 \%(n=1)$ showed reliable change on the GDS.

\section{Exploratory Analyses}

Within-subjects analyses of PES-E-20 and PA measures. Given the interesting pattern of results that were found on the repeated measures analyses for the HRSD and GDS, one-way repeated measures ANOVAs were also used to compare scores on both the PES-E-20 and PA 
measures at pre-treatment, post-treatment, and 1-month follow-up. Treatment outcome scores for both treatment conditions were combined for these analyses. There was a significant main effect for time on the PES-E-20, $F(2,24)=6.62, p=.01$, multivariate partial eta squared $=.36$.

Exploration of the means showed that pleasant events on the PES-E-20 were rated higher at both post-treatment $(M=55.96, S D=9.41)$ and 1-month follow-up $(M=56.54, S D=9.86)$ than they were at pre-treatment $(M=52.08, S D=11.81)$. However, post-hoc comparisons using Fisher's LSD demonstrated that pre-treatment to 1-month follow-up was the only time period in which there was significant difference, $p=.001$. Effect size for this difference was small $(d=.40)$. A main effect for time was not significant for the PA scale, $F(2,24)=2.74, p=.09$, multivariate partial eta squared $=.19$. Please refer to Table 6 to review the mean scores used for the withinsubjects analyses on the PES-E-20 and PA and Figure 6 for a graphical representation of these scores.

Between-subjects 'completers only' analyses. A series of one-way between-groups analyses of covariance (ANCOVAs) were conducted to examine the main hypotheses and Research Question 1 using data from those who completed the full treatment program only Table 6

Pre-and Post-treatment and 1-month Follow-up Means for Within-Subjects ANOVAs

\begin{tabular}{|c|c|c|c|c|c|c|}
\hline \multirow[b]{2}{*}{ Variable } & \multicolumn{2}{|c|}{ Pre-treatment } & \multicolumn{2}{|c|}{$\underline{\text { Post-treatment }}$} & \multicolumn{2}{|c|}{$\underline{1 \text {-month follow-up }}$} \\
\hline & $M$ & $S D$ & $\bar{M}$ & $S D$ & $M$ & $S D$ \\
\hline PES-E-20 & 52.08 & 11.81 & 55.96 & 9.41 & 56.54 & 9.86 \\
\hline PA & 31.42 & 6.73 & 34.19 & 6.78 & 34.00 & 5.87 \\
\hline
\end{tabular}

Note. PES-E-20 = Pleasant Events Scale-Elderly-20; PA = Positive Affect Scale of the Positive and Negative Affect Schedule. 


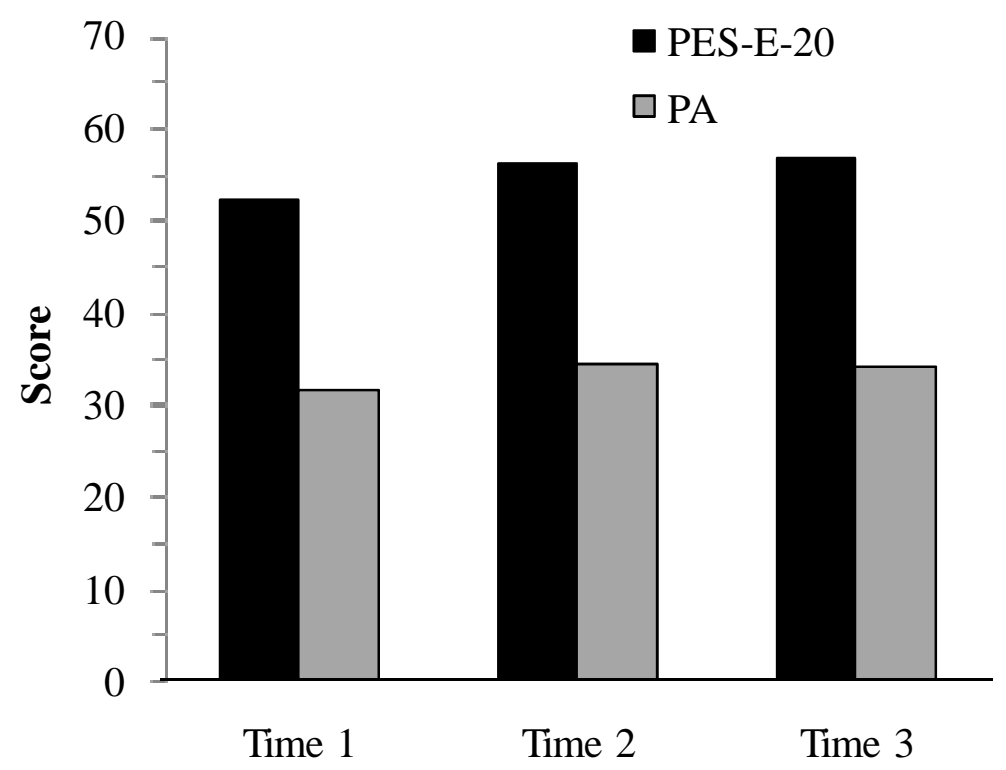

Figure 6. Significant within-subjects main effects for Time on the PES-E-20, but not the PA scale. $\mathrm{T} 1=$ pre-treatment, $\mathrm{T} 2=$ post-treatment, and $\mathrm{T} 3=1$-month follow-up for these analyses. PES-E-20 T1 to T3 is significantly higher, $p=.01$. All other findings non-significant.

(i.e., did not discontinue participation early). These analyses were conducted to compare scores on the HRSD, GDS, PA, and PES-E-20 between the immediate intervention and delayed treatment control groups at assessment T2 (i.e., following treatment for the immediate group and following delay for the control group). The pattern of results in these analyses was similar to those yielded in the analyses of the total sample. After adjusting for pre-intervention scores, there was a significant difference between the two treatment groups on T2 HRSD scores, $F(1,15)=$ $10.61, p=.01$, partial eta squared $=.41$. The T1 HRSD covariate explained $42 \%$ of the variance in T2 HRSD scores, partial eta squared $=.42$. Post-hoc visual comparison of the raw means suggested that depressive symptoms on the HRSD were significantly lower at T2 for the immediate treatment group $(M=3.80, S D=4.24)$ than they were for the delayed treatment control group $(M=8.38, S D=3.70)$. The effect size of this difference was large; $d=1.15$. ANCOVAs of the other dependent variables were not significant when controlling for baseline 
scores; GDS: $F(1,15)=.39, p=.54$, partial eta squared $=.03$, PA: $F(1,15)=1.17, p=.30$, partial eta squared $=.07$, PES-E-20: $F(1,15)=.548, p=.47$, partial eta squared $=.04$. It should be noted that the GDS analysis violated the assumption of equality of variance.

Within-subjects 'completers-only' analyses on depression measures. Treatment outcome scores for both treatment conditions were combined into one database for these analyses. Oneway repeated measures ANOVAs were used to examine Research Questions 2 and 3 using data from only those who completed the full treatment program. Scores on both the HRSD and GDS were compared at pre-treatment, post-treatment, and 1-month follow-up for only those participants who completed the study $(n=18)$. Findings from these analyses were similar to those found in the within-subjects analyses of the entire sample. There was a significant main effect for time on the HRSD, $F(2,16)=20.60, p<.001$, multivariate partial eta squared $=.72$. Post-hoc comparisons using Fisher's LSD suggested that depressive symptoms on the HRSD were rated significantly lower at both post-treatment $(M=2.67, S D=3.53, p<.001)$ and 1 month follow-up $(M=4.11, S D=6.47, p<.001)$ than they were at pre-treatment $(M=10.00, S D$ $=6.13)$. Effect sizes for these differences were both large, $d=1.43$ and .91 , respectively. As with the total sample analyses, scores on the HRSD were rated significantly higher at 1-month followup than they were at post-treatment, $p=.05$. A significant main effect for time was also found on the GDS, $F(2,16)=5.82, p=.01$, multivariate partial eta squared $=.42$. Post-hoc comparisons using Fisher's LSD suggested that depressive symptoms on the GDS were not rated significantly lower at post-treatment $(M=6.94, S D=4.70)$ than they were at pre-treatment $(M=8.44, S D=$ $5.97), p=.23$. However, scores on the GDS were significantly lower at 1-month follow-up ( $M=$ 5.83, $S D=4.76$ ) than they were at pre-treatment, $p=.01$. The effect size of this difference was 
medium, $d=.50$. Although scores on the GDS were lower at 1-month follow-up than they were at post-treatment, this result was not significant, $p=.08$.

Symptom change on the HRSD. Given the disparate findings on the HRSD and GDS for the main hypotheses, an exploratory analysis was conducted examining somatic (e.g., insomnia, headaches, hyperventilation, fatigue) versus affective (e.g., helpless, hopeless, worthless, guilty) symptoms on the HRSD, as this is a difference between the two measures that has been identified in the literature (e.g., Yesavage et al., 1983). The HRSD was divided into two separate subscales post-hoc, and ANCOVAs were conducted examining between-group differences on T2 somatic and affective symptoms while controlling for T1 scores. A significant difference was found between the immediate $(M=3.85, S D=3.00)$ and delayed $(M=7.38, S D=3.02)$ treatment groups on somatic symptoms at $\mathrm{T} 2, F(1,23)=14.73, p=.001$, partial eta squared $=$ .39. The effect size of this difference was large, $d=1.14$. The T1 somatic covariate explained $40 \%$ of the variance in T2 somatic scores, partial eta squared $=.40$. However, it is important to note that the assumption of equality of variances was not meet for this analysis. Nevertheless, this finding was replicated when examining data from only those participants who completed the study. In this analysis, the assumption of equality of variances was met. For 'completers-only,' mean somatic scores for the immediate group $(M=2.70, S D=2.36)$ were significantly lower than they were for the delayed treatment group $(M=6.25, S D=2.71)$ at $\mathrm{T} 2$ when controlling for T1 scores, $F(1,15)=19.02, p=.001$, partial eta squared $=.56$. The effect size of this difference was also large, $d=1.34$.

Affective symptoms as measured by the HRSD were not significantly different at T2 between the immediate $(M=1.62, S D=2.29)$ and delayed $(M=3.62, S D=2.40)$ treatment groups, $F(1,23)=4.07, p=.06$, partial eta squared $=.15$. This finding was also replicated when 
looking at 'completers-only' data. A significant difference was not found in HRSD affective symptoms between immediate $(M=1.10, S D=2.13)$ and delayed $(M=2.38, S D=1.69)$ treatment groups at $\mathrm{T} 2$ when controlling for $\mathrm{T} 1$ affective symptoms, $F(1,15)=1.86, p=.19$, partial eta squared $=.11$. Please refer to Figure 7 for a graphical display of these results.
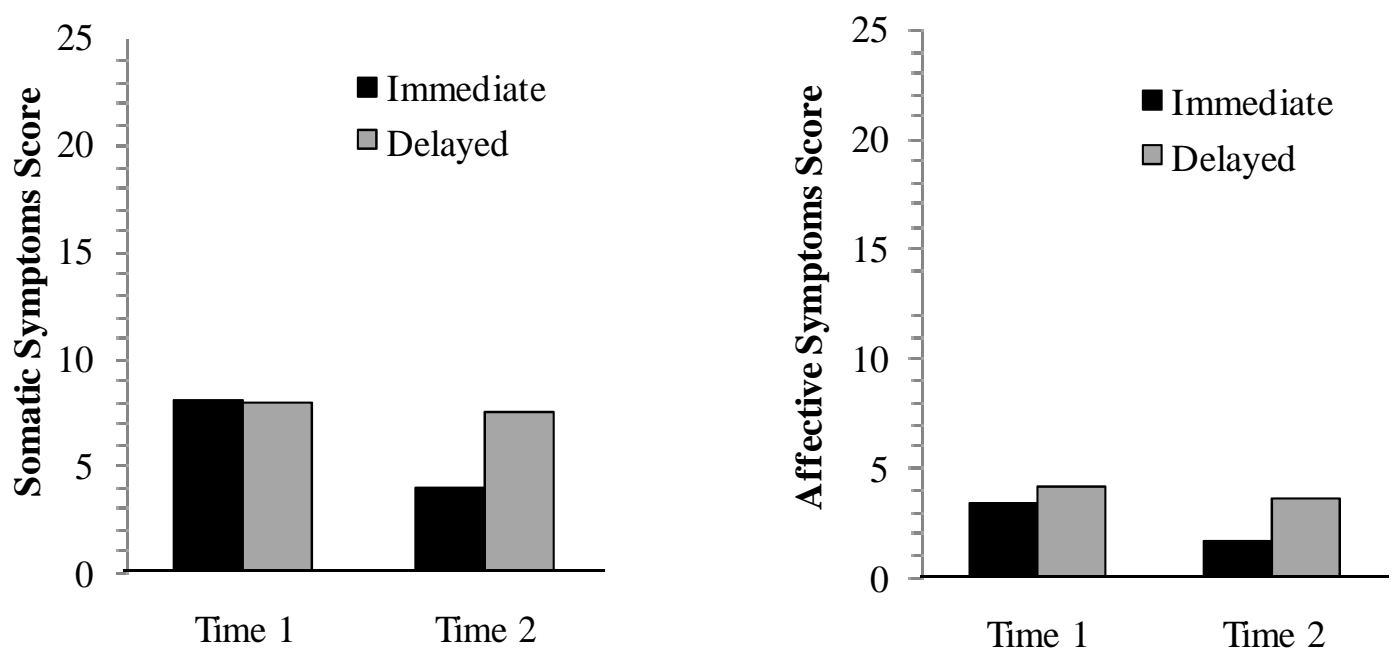

Figure 7. Somatic and affective symptoms at T1 and T2 on the HRSD. Experimental groups differed significantly at $\mathrm{T} 2$ on the somatic symptoms, but not on the affective symptoms.

Given the results noted above, it was questioned whether either of these two symptom patterns may have been related to the changes seen on the HRSD in the repeated measures analyses for Research Questions 2 and 3. Somatic and affective symptoms were again separated out for these analyses, and one-way repeated measures ANOVAs were used to compare somatic and affective scores on the HRSD at pre-treatment, post-treatment, and 1-month follow-up. There was a significant main effect for time on both the somatic, $(F(2,24)=13.36, p<.001$, multivariate partial eta squared $=.53)$ and affective $(F(2,24)=6.94, p=.004$, multivariate partial eta squared $=.37$ ) symptoms. Post-hoc comparisons using Fisher's LSD demonstrated that somatic symptoms were rated significantly lower at both post-treatment $(M=4.08, S D=$ 
$3.77, p<.001)$ and 1-month follow-up $(M=4.81, S D=4.20, p<.001)$ than they were at pretreatment $(M=7.65, S D=3.29)$. This same pattern was found for affective symptoms; they were rated significantly lower at both post-treatment $(M=1.88, S D=2.67, p=.001)$ and 1-month follow-up $(M=2.15, S D=2.57, p=.004)$ than they were at pre-treatment $(M=3.46, S D=2.76)$. Neither symptom subtype was significantly different between post-treatment and 1-month follow-up, suggesting that neither somatic nor affective symptoms alone contributed more to the increase in HRSD scores at 1-month follow-up found in the repeated measures analyses for Research Questions 2 and 3.

\section{Treatment Implementation}

Treatment receipt. To assess treatment receipt, a paired-samples $t$-test was conducted on the BAKT collapsed across treatment groups to evaluate the impact of the intervention on participants' knowledge of BA principles from baseline to post-treatment. A statistically significant change in BAKT scores was not found between baseline $(M=21.85, S D=1.95)$ and post-treatment $(M=21.73, S D=2.46), t(25)=.36, p=.72$, indicating that the intervention did not have a significant impact on BAKT scores. In addition, separate simple linear regressions were conducted to determine whether the independent variable (residualized change scores on the BAKT) predicted a significant amount of the variance in the dependent variables (residualized change scores on both the HRSD and GDS). Results of these analyses suggested that change in participants' knowledge of BA principles did not predict a significant amount of the variance in their change in depressive symptoms on the HRSD, $R$ squared $=.02$, adjusted $R$ squared $=-.02, F=.60, p=.45$, or the GDS, $R$ squared $=.01$, adjusted $R$ squared $=-.04, F=$ $.13, p=.72$. 
Treatment enactment. Percentage of pages of self-help text read ranged from $0-100, M$ $=61.19 \%, S D=42.50$. Percentage of written exercises completed ranged from $0-97, M=$ $41.08 \%, S D=35.29$. Simple linear regression analyses were conducted to examine the relations between treatment enactment variables and residualized change in depressive symptoms on both the HRSD and GDS over the course of treatment. With residualized change on the HRSD as the dependent variable and percentage of pages read as the independent variable, $R$ squared $=.55$, adjusted $R$ squared $=.53, F=29.40, p<.001$, indicating that percentage of pages read accounted for approximately $53 \%$ of the variance of change on the HRSD. Percentage of written exercises completed accounted for approximately $46 \%$ of the variance of change on the HRSD, $R$ squared $=.48$, adjusted $R$ squared $=.46, F=22.29, p<.001$. Exploratory follow-up regression analyses showed that percentage of pages read and written exercises completed each separately significantly predicted change in both somatic and affective symptoms on the HRSD. Percentage of pages read accounted for approximately $55 \%$ of change in somatic symptoms, $R$ squared $=$ .56 , adjusted $R$ squared $=.55, F=31.11, p<.001$, and $42 \%$ of change in affective symptoms, $R$ squared $=.44$, adjusted $R$ squared $=.42, F=18.97, p<.001$. Percentage of exercises completed accounted for approximately $46 \%$ of change in somatic symptoms, $R$ squared $=.48$, adjusted $R$ squared $=.46, F=22.27, p<.001$, and $37 \%$ of change in affective symptoms, $R$ squared $=.39$, adjusted $R$ squared $=.37, F=15.55, p=.001$.

With residualized change on the GDS as the dependent variable and percentage of pages read as the independent variable, $R$ squared $=.14$, adjusted $R$ squared $=.10, F=3.82, p=.06$, indicating that percentage of pages read did not account for a significant amount of change on the GDS. Percentage of written exercises completed also did not account for a significant amount of variance of change on the GDS, $R$ squared $=.08$, adjusted $R$ squared $=.04, F=2.14, p=.16$. 


\section{Treatment Benefit}

Descriptive statistics were used to evaluate participants' reports of treatment satisfaction and benefit. These data were collected only for individuals who completed the project $(n=18)$. Overall, participants reported that the treatment was helpful in general, with $72 \%$ rating the general helpfulness of the treatment as a ' 6 ' or higher on a scale from $0-10(0=$ least helpful; 10 $=$ most helpful), $M=6.72, S D=3.12$, range $=0-10$. The same percentage rated the helpfulness of the readings as a ' 6 ' or greater also, $M=6.72, S D=3.43$, range $=0-10$. Thoughts regarding the helpfulness of the written exercises were mixed, with $50 \%$ rating them as a ' 6 ' or higher, $M=$ 5.22, $S D=3.35$, range $=0-10$. Participants tended to affirm that BA treatment in general was well-suited to people of their age, with $67 \%$ rating this domain as a ' 6 ' or more $(0=$ not wellsuited; $10=$ very well-suited), $M=7.50, S D=2.33$, range $=4-10$. Similarly, participants tended to report feeling the book was well-suited to people of their age, with $61 \%$ rating this domain as a '6' or greater, $M=7.00, S D=2.40$, range $=3-10$. Most participants $(72 \%)$ rated the reading difficulty of the book at ' 5 ' or below on a scale from $0-10(0=$ not difficult; $10=$ very difficult), $M=4.06, S D=3.28$, range $=0-10$. Finally, $89 \%$ of participants $(n=16)$ indicated that they would recommend the self-help book to a friend. Overall, these quantitative results suggest that participants felt that both the book and BA treatment in general were helpful and mostly applicable to older adults, though it appears that the opinions regarding written exercises were mixed.

Open-ended questions regarding positive and negative aspects of the project yielded additional information. Some participants indicated that they could not relate to the content due to not feeling depressed. Suggestions for improvement included more time to complete the project, specific examples in the book referring to older adults, and the incorporation of 
additional pictures in the self-help text. In terms of positive aspects of the project, participants expressed that it was "worthwhile," "makes you more aware," "a most helpful vehicle for a person trying to overcome depression," and "it makes sense to me where medicine does not." There was also a theme of increased knowledge following the program (e.g., "I learned to take small steps at first," "I learned how my tired feelings and procrastination were related to depression," and "I've learned I can live in the moment and can enjoy more hobbies."). 


\section{CHAPTER 4}

\section{DISCUSSION}

Overall, the results of this investigation suggest that a behavioral activation bibliotherapy treatment may be useful in treating mild or subthreshold depressive symptoms in an older adult population. Symptoms on a clinician-rated measure of depression were significantly lower at the T2 assessment period for those who received BA bibliotherapy compared with those who were in the delayed treatment control condition, lending support for Hypothesis 1. However, contrary to the prediction of Hypothesis 2, depressive symptoms on a self-report measure were not significantly different at $\mathrm{T} 2$ between treatment groups. The findings for both of these hypotheses were replicated in the exploratory analyses examining data from only those participants who completed the study.

In response to Research Question 1, pleasant events and positive affect were not significantly different between groups at T2, though mean scores for both variables were trending in an upward direction for those in the immediate treatment group, and in a downward direction for those in the delayed treatment group. As with the main hypotheses, similar results were found through the examination of data for only those participants who completed the study.

When data for both groups were combined and examined in the context of a withinsubjects design to answer Research Questions 2 and 3, scores on the clinician-rated depression measure were significantly lower from pre-treatment to both post-treatment and 1-month followup. Examination of clinical significance showed that symptom change from pre- to posttreatment on the clinician-rated measure was statistically reliable in $39 \%$ of participants who 
completed the program. However, scores on this measure significantly increased from posttreatment to 1-month follow-up. Interestingly, self-reported depressive symptoms consistently decreased over the course of the treatment and follow-up periods. Although self-reported symptoms did not decrease significantly from pre- to post-treatment, they continued to decrease to a degree that reached statistical significance at 1-month follow-up. The findings for both of these measures were replicated in the exploratory within-subjects examination of 'completersonly' data.

Exploratory within-subjects analyses were conducted to examine changes in pleasant events and positive affect throughout the course of treatment for the entire study sample. Similar to the findings with the self-report measure of depression, pleasant events moved in a positive direction, though not significantly, from pre- to post-treatment. However, participant report of pleasant event engagement and enjoyment continued to increase to a degree that reached statistical significance at 1-month follow-up. Participant report of positive affect, though it increased slightly from pre- to post-treatment and remained stable through 1-month follow-up, did not differ to a statistically significant degree across assessment periods.

Treatment implementation analyses showed that, despite completing a moderate amount of both reading and written exercises on average, participants' knowledge of BA principles remained relatively stable from baseline to post-treatment. However, it is more likely that the lack of improvement found on the Behavioral Activation Knowledge Test was due to a problem of measurement versus a true lack of understanding of the material presented in the self-help text. The BAKT was updated for use in this investigation without pilot testing or a priori psychometric evaluation. Also, the original measure from which it is based has not been 
psychometrically tested on an older adult population. Further, internal consistency was found to be very low on the updated measure when analyzed following this study.

Despite issues of measurement on the instrument that was intended to formally evaluate treatment receipt, there were some informal indicators that participants acquired knowledge about the treatment over the course of the investigation. For example, participants rated the reading difficulty of the text as low. Also, their comments on the treatment benefit measure seemed to reflect a general understanding of BA principles. Further, a significant amount of the variance in change in clinician-rated depression was explained by the amount of pages of selfhelp text read and written exercises completed, and the amount of variance in change on the GDS was approaching significance for amount of pages read. This finding seems to suggest that the more bibliotherapy treatment a participant received via engagement with the study materials, the more changes were seen in depressive symptoms. Based on these findings, it would seem logical to speculate that the relation between treatment enactment and change in depressive symptoms was mediated by an increased understanding of treatment principles. However, as this speculation cannot be tested with the data collected for this study, the nature of the relationship between these variables remains unclear.

The incongruent results found between clinician-rated and self-reported symptoms for the main hypotheses are admittedly puzzling, particularly given that the Hamilton Rating Scale for Depression and Geriatric Depression Scale have demonstrated adequate concurrent validity and also that these measures were highly positively correlated at baseline. Exploratory follow-up analyses were conducted examining the known difference between the two measures. Specifically, the HRSD emphasizes somatic symptoms associated with depression to a greater degree than does the GDS. Conversely, the GDS focuses to a greater degree on affective 
symptoms associated with depression. When these symptom patterns were analyzed separately, somatic symptoms differed significantly by treatment group at $\mathrm{T} 2$, but affective symptoms did not. The reasons behind these differential findings are unclear. One possible explanation for this is that BA may be better at targeting the somatic symptoms of depression than the more affective symptoms of the disorder. In the primary investigator's aforementioned small $n$, multiple baseline investigation of face-to-face BA as a treatment for below average quality of life in activity-limited older adults, one of the major findings was that scores on a scale measuring distress related to somatic complaints dropped nearly nine $\mathrm{T}$ score points, or nearly a full standard deviation, on average over the course of the treatment. The ability of BA to better treat the somatic symptoms of depression than the affective symptoms remains an empirical question. However, if this is the case, it may provide an explanation as to why the treatment has been shown in the literature to be successful in treating those who do not respond well to cognitive therapy, which emphasizes clients' examination of the affective symptoms of depression to a greater degree than it does somatic symptoms.

Another possible explanation for the discrepant findings between somatic and affective symptoms involves assertions in the literature regarding older adults' differential symptom presentation of depressive disorders. As older adults are likely to present with more somatic than affective complaints of depression, they may be more attuned to changes in somatic symptoms that occur during depression treatment. However, this contention is offered with much circumspection, as older adults in other intervention studies for late life depression have reported significant decreases in affective symptoms following treatment.

Due to the present study's sample size and the exploratory nature of the analyses related to patterns of symptom change, the results regarding somatic and affective symptoms should be 
interpreted with caution. These results are tenuous particularly because when change on the HRSD was examined over repeated time periods across the total study sample, somatic and affective symptoms did not evidence disparate results (i.e., they both decreased from pretreatment to post-treatment and increased from post-treatment to 1-month follow-up together). In fact, further exploratory treatment enactment analyses showed that a significant amount of the variance of change in both affective and somatic symptoms on the HRSD from pre- to posttreatment were predicted by percent completion of the readings and written exercises. However, change in somatic symptoms was predicted to a greater degree by these variables.

Further, it is important to note that depressive symptoms on the GDS decreased to a significant degree at 1-month follow-up, indicating that the affective symptoms of depression did eventually decrease per participants' self-report. This change could possibly be explained by the passage of time alone. However, the intervention period timeline could also have played a role in delaying self-reported change in depressive symptoms. For example, during the last few days of the intervention phase, participants were asked to begin identifying short and long-term behavioral goals to improve mood along with a plan in which to implement those goals. This left little, if any, time for participants to act on these plans prior to completing the post-treatment assessment (which occurred within a week of intervention phase completion). This may also explain why pleasant events did not increase significantly until 1-month follow-up. A similar pattern was found on self-reported depression by Floyd and colleagues (2004) in their investigation comparing cognitive bibliotherapy to individual cognitive therapy. At the posttreatment phase of their study, individual psychotherapy conferred a significant advantage over the 4-week bibliotherapy intervention on self-reported depression. However, self-reported 
depression continued to improve following treatment, resulting in non-significant differences between treatments at 3-month follow-up (Floyd et al., 2004).

However, these findings do not explain why symptoms on the HRSD did not improve, and in fact increased, from post-treatment to 1-month follow-up. In the Floyd et al. (2004) investigation, HRSD scores also continued to improve between post-treatment and 3-month follow-up. As it is challenging to conceive of a clinical or theoretical reason for this change, it is probably instead related to methodological shortcomings of the study, which will be described in the subsequent section. It is important to keep in mind, however, that despite the significant increase in HRSD scores from post-treatment to 1-month follow-up, 1-month follow-up scores on the clinician-rated measure were significantly lower than those at pre-treatment.

\section{Study Limitations}

This investigation has a number of limitations. The generalizability of this study is questionable, as the sample consisted of predominantly White, female participants who were recruited from two very specific locations. In other words, the sample cannot be described as a random and representative sample of older adults in general. Also, depressive symptoms were near or within normal limits for most of the sample at baseline, so it is difficult to make clear assertions about the ability of the BA bibliotherapy treatment to treat anything other than mild or subthreshold symptomatology. In fact, all findings from this study should be interpreted with caution, particularly those results that are exploratory and examined only subsets of the full study sample. Results must also be considered in light of the sample size of the investigation, which started out small and decreased with participant attrition. Readers should also note that following the post-treatment assessment period for the immediate treatment group, the research 
investigation was no longer controlled. Thus, results from the repeated measures analyses are based on uncontrolled research data.

Further, there were some procedural limitations that may have had an impact on the validity of the study. Participant compliance with treatment was moderate on average. Only $35 \%$ of participants completed $100 \%$ of the readings per their self-report, and none of the participants completed $100 \%$ of the written exercises. It is challenging to make any definitive claims as to the efficacy of the intervention when participants did not engage in the full course of treatment. This also points to another limitation, that participants could have benefitted from more time to complete the treatment program. Due to the amount of time available to complete this study, participants were allotted only four weeks to read the self-help book and complete the written exercises therein. However, the authors of the self-help text have suggested that eight weeks is the ideal length of time to complete the readings and written exercises in the self-help text (which would allow for completion of roughly one chapter per week) in order for individuals to benefit most from the program. If study participants would have had more time to engage with study materials, they likely would have had additional time to work on implementing short- and long-term behavioral goals to improve mood.

Issues of measurement were another limitation of this investigation. One threat to the validity of the study involved the administration of the HRSD at the Time 2 assessment period. This project had virtually no budget and, thus, research assistants were available on a strictly voluntary basis. As it is generally recommended that the clinician-rated depression measure be administered by an experienced clinician, advanced graduate students from the researcher's laboratory completed these assessments. However, due to the time constraints of these individuals' schedules, an undergraduate research assistant completed the remainder of the 
assessments at T2. Further, due to differential availability of these voluntary assistants, it was not uncommon for HRSDs to be completed on a day other than the day in which the remainder of the assessment battery was administered. Clearly, it would have been preferable to have one individual complete the entire T2 assessment battery on the same day. Underscoring these obvious threats to validity are the inter-rater reliability findings on the HRSD. Although the $88 \%$ agreement that was attained during this study is certainly acceptable, it is lower than the $94 \%$ agreement rate reached prior to the study as well as the $95 \%$ agreement rate that was found in the study of the GRID-HAMD (the grid procedure used to score the HRSD). It is therefore possible that a mild degree of rater drift could have occurred over the course of the study. Also, as previously indicated, participants were not instructed to refrain from revealing their experimental condition to interviewers, introducing a potential confound into the investigation. Despite this, when questioned after the investigation, interviewers reported that they could not recall an instance of a participant revealing their treatment condition. Nevertheless, this should be kept in mind. Further, as mentioned previously, the lack of an established measure with which to measure treatment receipt was also problematic.

\section{Future Directions}

Subsequent studies examining BA bibliotherapy as a treatment for late life depressive symptoms may consider employing a larger sample size comprised of individuals that are more representative of the general older adult population. It would also be beneficial for future researchers in this realm to test Overcoming Depression One Step at a Time in a sample that is experiencing a greater level of severity of depressive symptoms. Further, as stated previously, participants in future studies would likely benefit from increased time to complete the treatment program to improve the likelihood of increased treatment compliance. In addition, the 
incorporation of additional follow-up periods would prove useful in determining the ability of the treatment to maintain or improve symptoms long-term.

Additional studies in this area could include controlled comparisons of BA bibliotherapy and cognitive bibliotherapy. Given the results from the current study, it would be interesting to determine whether either treatment is more or less effective in treating somatic and affective symptoms. Furthermore, a study comparing the outcomes of younger and older adults following behavioral activation bibliotherapy would be a meaningful addition to the literature. In addition to treatment outcome research, the field would benefit from the development of instruments measuring BA-specific outcomes (e.g., avoidance and activation, pleasure and mastery, etc.). Summary and Conclusions

The present investigation extends the literature on behavioral activation treatment for older adults. It also provides very preliminary findings regarding the efficacy of BA bibliotherapy as a treatment for depressive symptoms in this population. Although additional research is needed to clarify how robust this treatment is, it was demonstrated in this study that participants who received BA bibliotherapy evidenced significantly fewer depressive symptoms relative to controls at post-treatment as rated by interviewers. Findings also suggest that the treatment may potentially be helpful in reducing depressive symptoms and increasing pleasant events in older adults with mild or subthreshold symptoms of depression, particularly after given adequate time to engage with program materials. This treatment was generally well received by the participants in this study, and no one described it as detrimental or aversive. However, until more evidence is gathered regarding the efficacy of BA bibliotherapy, practitioners may still prefer to recommend cognitive bibliotherapy as a first line of treatment for appropriate individuals with late life mild-to-moderate depressive symptoms given its established evidence 
base. Despite this, there is no reason to suggest that BA bibliotherapy would not be useful for older adults who are feeling somewhat down and disengaged from normal routines.

Overall, the results indicate that BA bibliotherapy may be a promising treatment for older adults. It is expected that, with continued refinements of studies in this area and possible modifications to make the treatment more applicable to older adults, BA bibliotherapy can show even more potential for individuals in this age group. 


\section{REFERENCES}

Ackerson, J., Scogin, F., McKendree-Smith, N., \& Lyman, R. D. (1998). Cognitive bibliotherapy for mild and moderate adolescent depressive symptomatology. Journal of Consulting and Clinical Psychology, 66(4), 685-690.

Addis, M. E., \& Martell, C. R. (2004). Overcoming Depression One Step at a Time. Oakland, CA: New Harbinger Publications, Inc.

American Psychological Association Presidential Task Force on Evidence-Based Practice. (2006). Evidence-based practice in psychology. American Psychologist, 61, 271-285.

Areán, P. A., Uncapher, H., \& Satre, D. (1998). Depression. In M. Hersen \& V. B. Van Hasselt (Eds.), Handbook of Clinical Geropsychology (pp. 195-216). New York: Plenum Press.

Beck, A. T., Ward, C., Mendelsohn, M., Mock, J., \& Erbaugh, J. (1961). An inventory for measuring depression. Archives of General Psychiatry, 4, 53-63.

Bilich, L. L., Deane, F. P., Phipps, A. B., Barisic, M., \& Gould, G. (2008). Effectiveness of bibliotherapy self-help for depression with varying levels of telephone helpline support. Clinical Psychology \& Psychotherapy, 15(2), 61-74.

Birrer, R. B., \& Vemuri, S. P. (2004). Depression in later life: A diagnostic and therapeutic challenge. American Family Physician, 69(10), 2375-2382.

Blazer, D. (2002). Depression in late life ( $3^{\text {rd }}$ Ed.). New York: Springer.

Bottonari, K. A., Roberts, J. E., Thomas, S. N., Schmaling, K. B., \& Read, J. P. (2008). Stop thinking and start doing: Switching from cognitive therapy to behavioral activation in a 
case of chronic treatment-resistant depression. Cognitive and Behavioral Practice, 15, 376-386.

Bruce, M. L., McAvay, G. J., \& Raue, P. J. (2002). Major depression in elderly home health care recipients. American Journal of Psychiatry, 159, 1367-1374.

Burns, D. (1980). Feeling Good. New York: Signet.

Choi, N. G., \& Morrow-Howell, N. (2007). Low-income older adults' acceptance of depression treatments: Examination of within-group differences. Aging \& Mental Health, 11(4), 423-433.

Coffman, S. J., Martell, C. R., Dimidjian, S., Gallop, R., \& Hollon, S. D. (2007). Extreme nonresponse in cognitive therapy: Can behavioral activation succeed where cognitive therapy fails? Journal of Consulting and Clinical Psychology, 75(4), 531-541.

Cohen, J. (1988). Statistical Power Analysis for the Behavioral Sciences. (2 ${ }^{\text {nd }}$ Ed.), Hillsdale, NJ: Lawrence Erlbaum Associates.

Cuijpers, P., van Straten, A., \& Smit, F. (2006). Psychological treatment of late-life depression: A meta-analysis of randomized controlled trials. International Journal of Geriatric Psychiatry, 21(12), 1139-1149.

den Boer, P. C. A. M., Wiersma, D., \& Van Den Bosch, R. J. (2004). Why is self-help neglected in the treatment of emotional disorders? A meta-analysis. Psychological Medicine, 34(6), 959-971.

DeRubeis, R. J., Gelfand, L., Tang, T., \& Simons, A. D. (1999). Medication versus cognitive behavior therapy for severely depressed outpatients: Meta-analysis of four randomized comparisons. American Journal of Psychiatry, 156, 1007-1013. 
Dimidjian, S., Hollon, S. D., Dobson, K. S., Schmaling, K. B., Kohlenberg, R. J., Addis, M. E., et al. (2006). Randomized trial of behavioral activation, cognitive therapy, and antidepressant medication in the acute treatment of adults with major depression. Journal of Consulting and Clinical Psychology, 74(4), 658-670.

Dobson, K. S., Hollon, S. D., Dimidjian, S., Schmaling, K. B., Kohlenberg, R. J., Gallop, R. J., et al. (2008). Randomized trial of behavioral activation, cognitive therapy, and antidepressant medication in the prevention of relapse and recurrence in major depression. Journal of Consulting and Clinical Psychology, 76(3), 468-477.

Engels, G., \& Vermey, M. (1997). Efficacy of nonmedical treatments of depression in elders: A quantitative analysis. Journal of Clinical Geropsychology, 31, 17-35.

Ertan, F. S., Ertan, T., Kiziltan, G., \& Uygucgil, H. (2005). Reliability and validity of the Geriatric Depression Scale in depression in Parkinson's disease. Journal of Neurology, Neurosurgery, \& Psychiatry, 76(10), 1445-1447.

Ferster, C. B. (1973). A functional analysis of depression. American Psychologist, 28(10), 857870.

Flesch, R. (1951). How to Test Readability. New York: Harper.

Floyd, M. (2003). Bibliotherapy as an adjunct to psychotherapy for depression in older adults. Psychotherapy in Practice, 59(2), 187-195.

Floyd, M., Rohen, N., Shackleford, J. A. M., Hubbard, K. L., Parnell, M. B., Scogin, F., et al. (2006). Two-year follow-up of bibliotherapy and individual cognitive therapy for depressed older adults. Behavior Modification, 30(3), 281-294. 
Floyd, M., Scogin, F., \& Chaplin, W. F. (2004). The Dysfunctional Attitudes Scale: Factor structure, reliability, and validity with older adults. Aging \& Mental Health, 8(2), 153160.

Floyd, M., Scogin, F., McKendree-Smith, N. L., Floyd, D. L., \& Rokke, P. D. (2004). Cognitive therapy for depression: A comparison of individual psychotherapy and bibliotherapy for depressed older adults. Behavior Modification, 28(2), 297-318.

Gortner, E. T., Gollan, J. K., Dobson, K. S., \& Jacobson, N. S. (1998). Cognitive-behavioral treatment for depression: Relapse prevention. Journal of Consulting and Clinical Psychology, 66(2), 377-384.

Gottfries, C. G. (1998). Is there a difference between elderly and younger patients with regard to the symptomatology and aetiology of depression? International Clinical Psychopharmacology, 13(Suppl. 5), S13-S18.

Gregory, R., Canning, S., Lee, T., \& Wise, J. (2004). Cognitive bibliotherapy for depression: A meta-analysis. Professional Psychology: Research and Practice, 35(3), 275-280.

Gum, A. M., Areán, P. A., Hunkeler, E., Tang, L., Katon, W., Hitchcock, P., et al. (2006). Depression treatment preferences in older primary care patients. Gerontologist, 46(1), 1422.

Guy, W. (1976). ECDEU Assessment Manual for Psychopharmacology. Washington, DC: U.S. Department of Health, Education, and Welfare.

Hamilton, M. (1967). Development of a rating scale for primary depressive illness. British Journal of Social \& Clinical Psychology, 6(4), 278-296.

Harris, Y., \& Cooper, J. K. (2006). Depressive symptoms in older people predict nursing home admission. Journal of the American Geriatrics Society, 54(4), 593-597. 
Hertzog, C., \& Hultsch, D. F. (2000). Metacognition in adulthood and old age. In Craik, F. I. M., \& Salthouse, T. A. (Eds.), The Handbook of Aging and Cognition (2 ${ }^{\text {nd }}$ Ed.). Mawah, NJ: Lawrence Erlbaum Associates Publishers.

Hirschfeld, R. M., Keller, M. B., Panico, S., Arons, B. S., Barlow, D., Davidoff, F., et al. (1997). The National Depressive and Manic-Depressive Association consensus statement on the undertreatment of depression. Journal of the American Medical Association, 277, 333340.

Hollon, S. D. (2000). Do cognitive change strategies matter in cognitive therapy? Prevention and Treatment, 3, Article 25.

Hollon, S. D., Jarrett, R. B., Nierenberg, A. A., Thase, M. E., Trivedi, M., \& Rush, A. J. (2005). Psychotherapy and medication in the treatment of adult and geriatric depression: Which monotherapy or combined treatment? Journal of Clinical Psychiatry, 66(4), 455-468.

Hopko, D. R., Lejuez, C. W., Ruggiero, K. J., \& Eifert, G. H. (2003). Contemporary behavioral activation treatments for depression: Procedures, principles, and progress. Clinical Psychology Review, 23(5), 699-717.

Hoyl, M. T., Alessi, C. A., Harker, J. O., Josephson, K. R., Pietruszka, F. M., Koelfgen, M., et al. (1999). Development and testing of a five-item version of the Geriatric Depression Scale. Journal of the American Geriatrics Society, 47(7), 873-878.

Jacobson, N. S., Dobson, K. S., Truax, P. A., Addis, M. E., Koerner, K., Gollan, J. K., et al. (1996). A component analysis of cognitive-behavioral treatment for depression. Journal of Consulting and Clinical Psychology, 64(2), 295-304. 
Jacobson, N. S., Martell, C. R., \& Dimidjian, S. (2001). Behavioral activation treatment for depression: Returning to contextual roots. Clinical Psychology: Science \& Practice, 8(3), 255-270.

Jacobson, N. S. \& Truax, P. (1991). Clinical significance: A statistical approach to defining meaningful change in psychotherapy research. Journal of Consulting and Clinical Psychology, 59(1), 12-19.

Jamison, C., \& Scogin, F. (1995). The outcome of cognitive bibliotherapy with depressed adults. Journal of Consulting and Clinical Psychology, 63(4), 644-650.

Jeste, D. V., Blazer, D. G., \& First, M. (2005). Aging-related diagnostic variations: Need for diagnostic criteria appropriate for elderly psychiatric patients. Biological Psychiatry, $58(4), 265-271$.

Jones, R. N., Marcantonio, E. R., \& Rabinowitz, T. (2003). Prevalence and correlates of recognized depression in U. S. nursing homes. Journal of the American Geriatrics Society, 51(10), 1404-1409.

Landreville, P. (1998). Cognitive bibliotherapy for depression in older adults with a disability. Clinical Gerontologist, 19(3), 69-75.

Landreville, P., Landry, J., Baillargeon, L., Guérette, A., \& Matteau, E. (2001). Older adults' acceptance of psychological and pharmacological treatments for depression. Journal of Gerontology: Psychological Sciences, 56B(5), 285-291.

Lewinsohn, P. M., \& Graf, M. (1973). Pleasant activities and depression. Journal of Consulting and Clinical Psychology, 41(2), 261-268.

Lewinsohn, P., Munoz, R., Youngren, M., \& Zeiss, A. (1986). Control Your Depression. Englewood Cliffs, NJ: Prentice-Hall. 
Lichstein, K. L., Riedel, B. W., \& Grieve, R. (1994). Fair tests of clinical trials: A treatment implementation model. Advances in Behaviour Research and Therapy, 16(1), 1-29.

Luppa, M., Heinrich, S., Angermeyer, M. C., König, H., \& Riedel-Heller, S. G. (2007). Cost-ofillness studies of depression: A systematic review. Journal of Affective Disorders, 98, 2943.

Lyons, J. S., Strain, J. J., Hammer, J. S., \& Ackerman, A. D. (1989). Reliability, validity, and temporal stability of the Geriatric Depression Scale in hospitalized elderly. International Journal of Psychiatry in Medicine, 19(2), 203-209.

Mains, J. A., \& Scogin, F. R. (2003). The effectiveness of self-administered treatments: A practice-friendly review of the research. Journal of Clinical Psychology, 59(2), 237-246.

Manthorpe, J., \& Iliffe, S. (2006). Suicide among older people. Nursing Older People, 17(10), 25-29.

Martell, C. R., Addis, M. E., \& Jacobson, N. S. (2001). Depression in context: Strategies for guided action. New York: W. W. Norton.

McKendree-Smith, N. L. (2000). Cognitive and behavioral bibliotherapy for depression: An examination of efficacy and mediators and moderators of change. Dissertation Abstracts International: Section B: The Sciences and Engineering, 60(11-B), 5783.

Mohr, D. C., Hart, S. L., Julian, L., Catledge, C., Honos-Webb, L., Vella, L., et al. (2005). Telephone-administered psychotherapy for depression. Archives of General Psychiatry, 62(9), 1007-1014.

Moss, K. (2007). The Behavioral Activation Knowledge Test. Unpublished manuscript, University of Alabama. 
Moss, K. S., \& Scogin, F. R. (2006). A quality of life intervention for older adults with perceived activity-limiting health problems using behavioral activation therapy. Unpublished master's thesis, University of Alabama, Tuscaloosa.

Moylan, T., Das, K., Gibb, A., Hill, A., Kane, A., Lee, C., et al. (2004). Assessment of cognitive function in older hospital inpatients: Is the Telephone Interview for Cognitive Status (TICS-M) a useful alternative to the Mini Mental State Examination? International Journal of Geriatric Psychiatry, 19(10), 1008-1009.

Naylor, E. V. (2008). A five-minute bibliotherapy prescription as a physician-delivered treatment for depression. Dissertation Abstracts International: Section B: The Sciences and Engineering, 69(4-B), 2635.

Ostir, G. V., Smith, P. M., Smith, D., \& Ottenbacher, K. J. (2005). Reliability of the Positive and Negative Affect Schedule (PANAS) in medical rehabilitation. Clinical Rehabilitation, 19(7), 767-769.

Pallant, J. (2001). SPSS Survival Manual. Philadelphia, PA: Open University Press.

Pinquart, M., \& Sorenson, S. (2001). How effective are psychotherapeutic and other psychosocial interventions with older adults? A meta-analysis. Journal of Mental Health and Aging, 7, 207-243.

Porter, J. F., Spates, C. R., \& Smitham, S. (2004). Behavioral activation group therapy in public mental health settings: A pilot investigation. Professional Psychology: Research and Practice, 35(3), 297-301.

Quality of Life and Older Rural Health Care Recipients (1 RO1 AG16311-01). Funded by NIA. Principal Investigator: Scogin, F. Co-Investigators: Burgio, L. \& Kaufman, A. 
Redding, R. E., Herbert, J. D., Forman, E. M., \& Guadiano, B. A. (2008). Popular self-help books for anxiety, depression, and trauma: How scientifically grounded and useful are they? Professional Psychology: Research and Practice, 39(5), 537-545.

Rivard, V. (2007). Perceived control and coping strategies in relation to anxious and depressive symptoms in women with activity restriction due to osteoarthritis. Dissertation Abstracts International: Section B: The Sciences and Engineering, 67, (10-B), 6075.

Rokke, P., \& Scogin, F. (1995). Depression treatment preferences in younger and older adults. Journal of Clinical Geropsychology, 1, 243-257.

Rule, B. G., Harvey, H. Z., \& Dobbs, A. R. (1989). Reliability of the Geriatric Depression Scale for younger adults. Clinical Gerontologist, 9(2), 37-43.

Rumsfeld, J. S., Jones, P. G., Whooley, M. A., Sullivan, M. D., Pitt, B., Weintraub, W. S., et al. (2005). Depression predicts mortality and hospitalization in patients with myocardial infarction complicated by heart failure. American Heart Journal, 150(5), 961-967.

Salamero, M., \& Marcos, T. (1992). Factor study of the Geriatric Depression Scale. Acta Psychiatrica Scandinavica, 86(4), 283-286.

Scogin, F. (1994). Assessment of depression in older adults: A guide for practitioners. In M. Storandt \& G. R. VandenBos (Eds.), Neuropsychological assessment of older adults: Dementia and depression. Washington, DC: American Psychological Association.

Scogin, F., Bynum, J., Stephens, G., \& Calhoon, S. (1990). Efficacy of self-administered treatment programs: Meta-analytic review. Professional Psychology: Research and Practice, 21(1), 42-47. 
Scogin, F., Floyd, M., Jamison, C., \& Ackerson, J. Landreville, P., \& Bissonnette, L. (1996). Negative outcomes: What is the evidence on self-administered treatments? Journal of Consulting and Clinical Psychology, 64(5), 1086-1089.

Scogin, F., Hamblin, D., \& Beutler, L. (1987). Bibliotherapy for depressed older adults: A selfhelp alternative. The Gerontologist, 27(3), 383-387.

Scogin, F., Jamison, C., \& Gochneaur, K. (1989). Comparative efficacy of cognitive and behavioral bibliotherapy for mildly and moderately depressed older adults. Journal of Consulting and Clinical Psychology, 57(3), 403-407.

Scogin, F., \& McElreath, L. (1994). Efficacy of psychosocial treatments for geriatric depression: A quantitative review. Journal of Consulting and Clinical Psychology, 62(1), 69-73.

Scogin, F., Rohen, N., \& Bailey, E. (2000). Geriatric Depression Scale. In M. E. Maruish (Ed.), Handbook of psychological assessment in primary care settings. Mahwah, NJ: Lawrence Erlbaum Associates.

Scogin, F., Welsh, D., Hanson, A., Stump, J., \& Coates, A. (2005). Evidence-based psychotherapies for depression in older adults. Clinical Psychology: Science and Practice, 12(3), 222-237.

Shanmugham, B., Karp, J., Drayer, R., Reynolds, C. F., \& Alexopoulos, G. (2005). Evidencebased pharmacologic interventions for geriatric depression. Psychiatric Clinics of North America, 28(4), 821-835.

Smith. M. L., \& Glass, G. V. (1977). Meta-analysis of psychotherapy outcome studies. American Psychologist, 32(9), 752-760. 
Snarski, S. (2007). The effectiveness of behavioral activation therapy with an inpatient geriatric psychiatry population. Unpublished doctoral dissertation, University of Alabama, Tuscaloosa.

Teri, L. \& Lewinsohn, P. M. (1982). Modification of the Pleasant and Unpleasant Events Schedules for use with the elderly. Journal of Consulting \& Clinical Psychology, 50(3), 444-445.

van Gool, C. H., Kempen, G. I., Penninx, B. W., Deeg, D. J., Beekman, A. T., \& van Eijk, J. T. (2005). Impact of depression on disablement in late middle aged and older persons: Results from the Longitudinal Aging Study Amsterdam. Social Science \& Medicine, $60(1), 25-36$.

Watson, D., Clark, L. A., \& Tellegen, A. (1988). Development and validation of brief measures of positive and negative affect: The PANAS scales. Journal of Personality and Social Psychology, 54(6), 1063-1070.

Watson, L. C., Garrett, J. M., Sloane, P. D., Gruber-Baldini, A. L., \& Zimmerman, S. (2003). Depression in assisted living: Results from a four-state study. American Journal of Geriatric Psychiatry, 11(5), 534-542.

Welsh, K. A., Breitner, J. C., \& Magruder-Habib, K. M. (1993). Detection of dementia in the elderly using telephone screening of cognitive status. Neuropsychiatry, Neuropsychology, \& Behavioral Neurology, 6(2), 103-110.

Westen, D., \& Morrison, K. (2001). A multidimensional meta-analysis of treatments for depression, panic, and generalized anxiety disorder: An empirical examination of the status of empirically supported therapies. Journal of Consulting and Clinical Psychology, 69(6), 875-899. 
Williams, J. B. (1988). A structured interview guide for the Hamilton Depression Rating Scale. Archives of General Psychiatry, 45, 742-747.

Williams, J. B., Kobak, K. A., Bech, P., Engelhardt, N., Evans, K., Lipsitz, J., et al. (2008). The GRID-HAMD: Standardization of the Hamilton Depression Rating Scale. International Clinical Psychopharmacology, 23, 120-129.

Williamson, G. M. (2000). Extending the activity restriction model of depressed affect: Evidence from a sample of breast cancer patients. Health Psychology, 19(4), 339-347.

Williamson, G. M. \& Schulz, R. (1992). Pain, activity, restriction, and symptoms of depression among community-residing elderly. Journal of Gerontology, 47, 367-372.

Williamson, G. M., Schulz, R., Bridges, M., \& Behan, A. (1994). Social and psychological factors in adjustment to limb amputation. Journal of Social Behavior and Personality, 9, 249-268.

Williamson, G. M. \& Shaffer, D. R. (2000). The activity restriction model of depressed affect: Antecedents and consequences of restricted normal activities. In G. M. Williamson, D. R. Shaffer, \& Parmelee, P. A. (Eds.), Physical illness and depression in older adults: A handbook of theory, research, and practice (pp. 173-200). Dordrecht, Netherlands: Kluwer Academic Publishers.

Yesavage, J. A., Brink, T. L., Rose, T. L., Lum, O., Huang, V., Adey, M. B., et al. (1983). Development and validation of a geriatric depression screening scale: A preliminary report. Journal of Psychiatric Research, 17, 37-49.

Yon, A., \& Scogin, F. (2009). Behavioral activation as a treatment for geriatric depression. Clinical Gerontologist, 32, 91-103. 
Zung, W. W. K. (1965). A self-rating depression scale. Archives of General Psychiatry, 12, 6370. 
APPENDIX 


\section{UNIVERSITY OF ALABAMA INSTITUTIONAL REVIEW BOARD FOR THE PROTECTION OF HUMAN SUBJECTS \\ REQUEST FOR APPROVAL OF RESEARCH INVOLVING HUMAN SUBJECTS}

I. Identifying information

$\begin{array}{rll} & \text { Principal Investigator } & \text { Second Investigator } \\ \text { Name: } & \text { Kathryn Moss } & \\ \text { Department: } & \text { Psychology } & \\ \text { College: } & \text { Arts \& Sciences } \\ \text { University: } & \text { University of Alabama } \\ \text { Address: } & \text { Box } 870348 \\ & \text { Tuscaloosa, AL 35487-0348 } \\ \text { Telephone: } & (205) 348-1921 \\ \text { FAX: } & \text { N/A } \\ \text { E-mail: } & \text { moss024@bama.ua.edu }\end{array}$

Title of Research Project: A Self-Help Behavioral Activation Treatment for Geriatric Depressive Symptoms

(Protocol \# 07-025)

Date Printed:

Funding Source: N/A

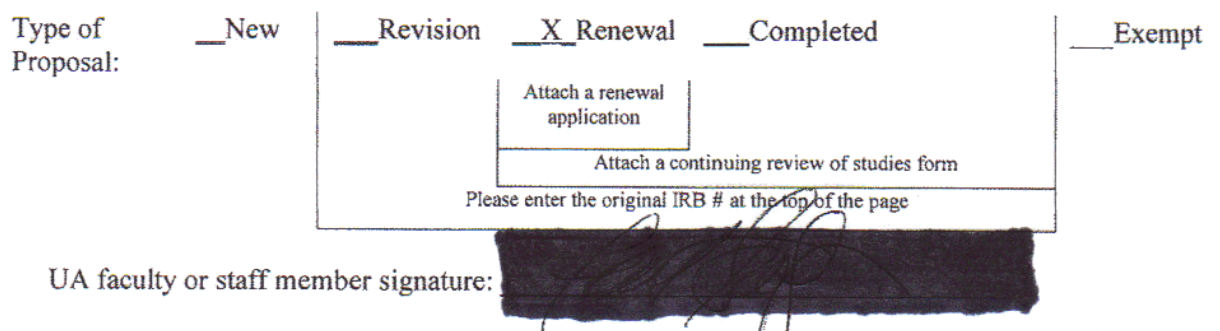

II. NOTIFICATION OF IRB ACTION (to be completed by IRB):

Type of Review: $\quad X$ Full board ___ Expedited

\section{IRB Action:}

Rejected

Tabled Pending Revisions

Approved Pending Revisions

$\chi_{\text {Approved - }}$ this proposal com Approval is effective until the following date: 1/30/2010 Items approved: Other:

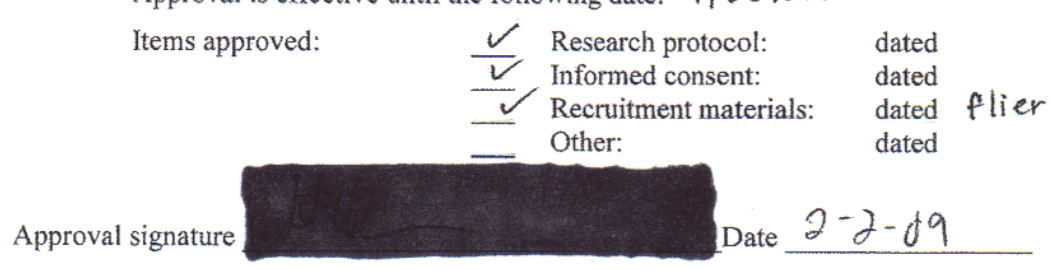

Date:

Date:

Date: 